\title{
Computation of Spectral Parameter of Discontinuous Dirac Systems with a Gaussian Multiplier
}

\author{
Mohammed M. Tharwat ${ }^{1,2}$ and Mohammed A. Alghamdi ${ }^{1}$ \\ ${ }^{1}$ Department of Mathematics, Faculty of Science, King Abdulaziz University, Jeddah 21589, Saudi Arabia \\ ${ }^{2}$ Department of Mathematics, Faculty of Science, Beni-Suef University, Beni-Suef 62511, Egypt
}

Correspondence should be addressed to Mohammed M. Tharwat; zahraa26@yahoo.com

Received 9 March 2014; Revised 21 April 2014; Accepted 21 April 2014; Published 13 May 2014

Academic Editor: Ali H. Bhrawy

Copyright (c) 2014 M. M. Tharwat and M. A. Alghamdi. This is an open access article distributed under the Creative Commons Attribution License, which permits unrestricted use, distribution, and reproduction in any medium, provided the original work is properly cited.

\begin{abstract}
We aim in this paper to apply a sinc-Gaussian technique to compute the eigenvalues of a Dirac system which has a discontinuity at one point and contains a spectral parameter in all boundary conditions. We establish the needed properties of eigenvalues of our problem. The error of this method decays exponentially in terms of the number of involved samples. Therefore the accuracy of the new technique is higher than the classical sinc-method. Numerical worked examples with tables and illustrative figures are given at the end of the paper.
\end{abstract}

\section{Introduction}

Problems with a spectral parameter in equations and boundary conditions form an important part of spectral theory of linear differential operators. A bibliography of papers in which such problems were considered in connection with specific physical processes can be found in $[1,2]$. Let $\sigma>0$. The Paley-Wiener space $\mathscr{B}_{\sigma}^{2}$ is the space of all entire functions of exponential type $\sigma$ which lie in $L^{2}(\mathbb{R})$ when restricted to $\mathbb{R}$, that is,

$$
\begin{gathered}
\mathscr{B}_{\sigma}^{2}:=\left\{f: f \text { entire, }|f(\mu)| \leq C e^{\sigma|\Im \mu|},\right. \\
\left.\int_{\mathbb{R}}|f(\mu)|^{2} d \mu<\infty\right\} .
\end{gathered}
$$

Assume that $f \in \mathscr{B}_{\sigma}^{2}$, and Whittaker-Kotel'nikov-Shannon sampling theorem (WKS) states that any function $f$ can be reconstructed via the classical sampling expansion, [3-5],

$$
f(\lambda)=\sum_{n \in \mathbb{Z}} f\left(\frac{n \pi}{\sigma}\right) \operatorname{sinc}(\sigma \lambda-n \pi), \quad \lambda \in \mathbb{C}
$$

where

$$
\operatorname{sinc}(\lambda):= \begin{cases}\frac{\sin (\lambda)}{\lambda}, & \lambda \neq 0 \\ 1, & \lambda=0 .\end{cases}
$$

In (2), the series is convergent uniformly on $\mathbb{R}$ and is convergent absolutely and uniformly on compact subsets of $\mathbb{C}$, cf. [6]. The WKS sampling series is widely used in approximation theory. It is used to approximate functions and their derivatives, solutions of differential and integral equations, and integral transforms (Fourier, Laplace, Hankel and Mellin) and to approximate the eigenvalues of boundary value problems; see, for example, [7-10]. The use of WKS sampling theory is called sinc methods, cf. [11-14]. The sincmethod has a slow rate of decay at infinity, which is as slow as $O\left(\left|\lambda^{-1}\right|\right)$. There are several attempts to improve the rate of decay. One of the interesting ways is to multiply the sincfunction in (2) by a kernel function, see, for example, [15-17]. Let $h \in(0, \pi / \sigma]$ and $\gamma \in(0, \pi-h \sigma)$. Assume that $\Phi \in \mathscr{B}_{\gamma}^{2}$ 
such that $\Phi(0)=1$, then for $f \in \mathscr{B}_{\sigma}^{2}$ we have the expansion, [18]

$$
f(\lambda)=\sum_{n=-\infty}^{\infty} f(n h) \operatorname{sinc}\left(h^{-1} \pi \lambda-n \pi\right) \Phi\left(h^{-1} \lambda-n\right) .
$$

The speed of convergence of the series in (4) is determined by the decay of $|\Phi(\lambda)|$. But the decay of an entire function of exponential type cannot be as fast as $e^{-c|x|}$ as $|x| \rightarrow \infty$, for some positive $c$, [18]. For $\sigma>0, h \in(0, \pi / \sigma]$, and $\omega:=$ $(\pi-h \sigma) / 2$, Schmeisser and Stenger, [18], defined the operator

$$
\begin{aligned}
\left(\mathscr{G}_{h, N} f\right)(\lambda):= & \sum_{n \in \mathbb{Z}_{N}(\lambda)} f(n h) \operatorname{sinc}\left(h^{-1} \pi \lambda-n \pi\right) \\
& \times G\left(\frac{\sqrt{\omega}(\lambda-n h)}{\sqrt{N} h}\right),
\end{aligned}
$$

where $G(t):=\exp \left(-t^{2}\right)$, which is called the Gaussian function, $\mathbb{Z}_{N}(\lambda):=\left\{n \in \mathbb{Z}:\left|\left[h^{-1} \Re \lambda+1 / 2\right]-n\right| \leq N\right\}$, $N \in \mathbb{N}$, and $[\lambda]$ denotes the integer part of $\lambda \in \mathbb{R}$, see also [19-21]. Note that the summation limits in (5) depend on the real part of $\lambda$. The authors, [18], proved that if $f$ is an entire function such that

$$
|f(\xi+i \eta)| \leq \phi(|\xi|) e^{\sigma|\eta|}, \quad \xi, \eta \in \mathbb{R},
$$

where $\phi$ is a nondecreasing, nonnegative function on $[0, \infty)$ and $\sigma \geq 0$, then for $h \in(0, \pi / \sigma), \omega:=(\pi-h \sigma) / 2, N \in \mathbb{N}$, and $|\Im \lambda|<N$, we have

$$
\begin{aligned}
\mid f(\lambda)- & \left(\mathscr{G}_{h, N} f\right)(\lambda) \mid \\
\leq & 2\left|\sin \left(h^{-1} \pi \lambda\right)\right| \phi(|\Re \lambda|+h(N+1)) \\
& \times \frac{e^{-\omega N}}{\sqrt{\pi \omega N}} \beta_{N}\left(h^{-1} \Im \lambda\right), \quad \lambda \in C,
\end{aligned}
$$

where

$$
\begin{aligned}
\beta_{N}(t):= & \cosh (2 \omega t)+\frac{2 e^{\omega t^{2} / N}}{\sqrt{\pi \omega N}\left[1-(t / N)^{2}\right]} \\
& +\frac{1}{2}\left[\frac{e^{2 \omega t}}{e^{2 \pi(N-t)}-1}+\frac{e^{-2 \omega t}}{e^{2 \pi(N+t)}-1}\right] .
\end{aligned}
$$

In [22], the authors derived an estimate for amplitude error: They proved that if

$$
\sup _{n \in \mathbb{Z}_{n}(\lambda)}|f(n h)-\tilde{f}(n h)|<\varepsilon
$$

holds, then for $|\Im \lambda|<N$, we have

$$
\left|\left(\mathscr{G}_{h, N} f\right)(\lambda)-\left(\mathscr{G}_{h, N} \tilde{f}\right)(\lambda)\right| \leq A_{\varepsilon, N}(\Im \lambda),
$$

where $\tilde{f}(n h)$ is the approximations of the exact values $f(n h)$ of (5), $\varepsilon>0$ is sufficiently small,

$$
A_{\varepsilon, N}(\Im \lambda)=2 \varepsilon e^{-\omega / 4 N}\left(1+\sqrt{\frac{N}{\omega \pi}}\right) \exp \left((\omega+\pi) h^{-1}|\Im \lambda|\right) .
$$

Consider the Dirac system which consists of the system of differential equations

$$
\begin{array}{r}
\left(\begin{array}{c}
\mathfrak{u}_{2}^{\prime}(x)-p_{1}(x) \mathfrak{u}_{1}(x) \\
\mathfrak{u}_{1}^{\prime}(x)+p_{2}(x) \mathfrak{u}_{2}(x)
\end{array}\right)=\lambda\left(\begin{array}{c}
\mathfrak{u}_{1}(x) \\
-\mathfrak{u}_{2}(x)
\end{array}\right), \\
x \in[-1,0) \cup(0,1],
\end{array}
$$

with boundary conditions

$$
\begin{aligned}
\mathscr{L}_{1}(\mathfrak{u}):= & \left(\alpha_{1}+\lambda \sin \theta_{1}\right) \mathfrak{t}_{1}(-1) \\
& -\left(\alpha_{2}+\lambda \cos \theta_{1}\right) \mathfrak{t}_{2}(-1)=0, \\
\mathscr{L}_{2}(\mathfrak{u}):= & \left(\beta_{1}+\lambda \sin \theta_{2}\right) \mathfrak{t}_{1}(1) \\
& -\left(\beta_{2}+\lambda \cos \theta_{2}\right) \mathfrak{t}_{2}(1)=0,
\end{aligned}
$$

and transmission conditions

$$
\begin{aligned}
& \mathscr{L}_{3}(\mathfrak{u}):=\mathfrak{u}_{1}\left(0^{-}\right)-\delta \mathfrak{u}_{1}\left(0^{+}\right)=0, \\
& \mathscr{L}_{4}(\mathfrak{u}):=\mathfrak{u}_{2}\left(0^{-}\right)-\delta \mathfrak{t}_{2}\left(0^{+}\right)=0 .
\end{aligned}
$$

Here $\lambda$ is the spectral parameter, $\mathfrak{t}=\left(\begin{array}{l}\mathfrak{u}_{1} \\ \mathfrak{u}_{2}\end{array}\right)$, the real valued function $p_{1}(\cdot)$ and $p_{2}(\cdot)$ are continuous in $[-1,0)$ and $(0,1]$ and have finite limits $p_{1}\left(0^{ \pm}\right):=\lim _{x \rightarrow 0^{ \pm}} p_{1}(x), p_{2}\left(0^{ \pm}\right):=$ $\lim _{x \rightarrow 0^{ \pm}} p_{2}(x) ; \alpha_{1}, \alpha_{2}, \beta_{1}, \beta_{2}, \delta \in \mathbb{R}, \theta_{1}, \theta_{2} \in[0, \pi)$, and $\delta \neq 0$. Throughout the following, we assume that

$$
\begin{aligned}
& \rho_{1}:=\alpha_{2} \sin \theta_{1}-\alpha_{1} \cos \theta_{1}>0, \\
& \rho_{2}:=\beta_{1} \cos \theta_{2}-\beta_{2} \sin \theta_{2}>0 .
\end{aligned}
$$

The aim of the present work is to compute the eigenvalues of (12)-(15) numerically by the sinc-Gaussian technique with errors analysis, truncation error, and amplitude error. In [23], Annaby and Tharwat computed the eigenvalues of a second-order operator pencil by sinc-Gaussian method. Also, the authors introduced some examples illustrating the sinc-Gaussian method accompanied by comparison with the sinc-method which explained that the sinc-Gaussian method gives remarkably better results; see also [24, 25]. Tharwat and Al-Harbi [26] computed the eigenvalues of discontinuous Dirac system but with eigenparameter in one boundary condition. In $[14,27]$ Tharwat et al. computed the eigenvalues of discontinuous Dirac system approximately, with eigenparameter appears in any of boundary conditions, by Hermite interpolations and regularized sinc-methods, respectively. In regularized sinc-method, also the same in Hermite interpolations method, the basic idea is that the eigenvalues are characterised as the zeros of an analytic function $f(\lambda)$ which can be written in the form $f(\lambda)=$ $k(\lambda)+u(\lambda)$, where $k(\lambda)$ is known part. The ingenuity of the approach is in trying to choose the function $f(\lambda)$ so that $u(\lambda) \in \mathscr{B}_{\sigma}^{2}$ (unknown part) and can be approximated by the sampling theorem if its values at some equally spaced points are known; see [11-14]. Recall that, in regularized sinc and Hermite interpolations methods, it is necessary that $u(\lambda)$ is $L^{2}$-function. In this paper we will use sinc-Gaussian sampling formula (5) to compute eigenvalues of (12)-(15) numerically. 
The proposed method reduces the error bounds remarkably (see examples of Section 4). The basic idea is to write the function of eigenvalues as the sum of two terms, one known and the other unknown but an entire function of exponential type which satisfies (6). In other words, the unknown term is not necessarily an $L^{2}$-function. Then we approximate the unknown part using (5) and obtain better results. We would like to mention that the papers in computing eigenvalues by sinc-Gaussian method are few; see [22-26]. In Sections 2 and 3, we discuss some properties of the eigenvalues of the boundary value problems (12)-(15) and also, we derive the sinc-Gaussian technique to compute the eigenvalues of (12)-(15) with error estimates. The last section involves some illustrative examples.

\section{Some Important Results}

In the following, we study some properties of the eigenvalues of the problems (12)-(15) which need in our method; see [26, $28]$. For functions $\mathfrak{t}(x)$, which defined on $[-1,0) \cup(0,1]$ and has finite limit $\mathfrak{u}( \pm 0):=\lim _{x \rightarrow \pm 0} \mathfrak{t}(x)$, by $\mathfrak{u}_{(1)}(x)$ and $\mathfrak{u}_{(2)}(x)$, we denote the functions

$$
\begin{aligned}
& \mathfrak{t}_{(1)}(x)= \begin{cases}\mathfrak{t}(x), & x \in[-1,0) ; \\
\mathfrak{u}\left(0^{-}\right), & x=0,\end{cases} \\
& \mathfrak{t}_{(2)}(x)= \begin{cases}\mathfrak{t}(x), & x \in(0,1] ; \\
\mathfrak{t}\left(0^{+}\right), & x=0,\end{cases}
\end{aligned}
$$

which are defined on $\Gamma_{1}:=[-1,0]$ and $\Gamma_{2}:=[0,1]$, respectively.

In the following lemma, we will prove that the eigenvalues of the problems (12)-(15) are real; see [29, 30].

Lemma 1. The eigenvalues of the problems (12)-(15) are real.

Proof. Assume the contrary that $\lambda_{0}$ is a nonreal eigenvalue of problems (12)-(15). Let $\left(\begin{array}{c}\mathfrak{u}_{1}(x) \\ \mathfrak{u}_{2}(x)\end{array}\right)$ be a corresponding (nontrivial) eigenfunction. By (12), we have

$$
\begin{array}{r}
\frac{d}{d x}\left\{\mathfrak{u}_{1}(x) \overline{\mathfrak{t}}_{2}(x)-\overline{\mathfrak{t}}_{1}(x) \mathfrak{t}_{2}(x)\right\} \\
=\left(\bar{\lambda}_{0}-\lambda_{0}\right)\left\{\left|\mathfrak{t}_{1}(x)\right|^{2}+\left|\mathfrak{t}_{2}(x)\right|^{2}\right\}, \\
x \in[-1,0) \cup(0,1] .
\end{array}
$$

Integrating the above equation through $[-1,0]$ and $[0,1]$, we obtain

$$
\begin{aligned}
\left(\bar{\lambda}_{0}-\right. & \left.\lambda_{0}\right)\left[\int_{-1}^{0}\left(\left|\mathfrak{u}_{1}(x)\right|^{2}+\left|\mathfrak{t}_{2}(x)\right|^{2}\right) d x\right] \\
= & \mathfrak{t}_{1}\left(0^{-}\right) \bar{u}_{2}\left(0^{-}\right)-\overline{\mathfrak{t}}_{1}\left(0^{-}\right) \mathfrak{u}_{2}\left(0^{-}\right) \\
& -\left[\mathfrak{u}_{1}(-1) \overline{\mathfrak{t}}_{2}(-1)-\overline{\mathfrak{t}}_{1}(-1) \mathfrak{u}_{2}(-1)\right],
\end{aligned}
$$

$$
\begin{aligned}
\left(\bar{\lambda}_{0}-\right. & \left.\lambda_{0}\right)\left[\int_{0}^{1}\left(\left|\mathfrak{t}_{1}(x)\right|^{2}+\left|\mathfrak{u}_{2}(x)\right|^{2}\right) d x\right] \\
= & \mathfrak{t}_{1}(1) \overline{\mathfrak{t}}_{2}(1)-\overline{\mathfrak{t}}_{1}(1) \mathfrak{u}_{2}(1) \\
& -\left[\mathfrak{u}_{1}\left(0^{+}\right) \overline{\mathfrak{t}}_{2}\left(0^{+}\right)-\overline{\mathfrak{t}}_{1}\left(0^{+}\right) \mathfrak{u}_{2}\left(0^{+}\right)\right] .
\end{aligned}
$$

Then from (13), (14), and transmission conditions, we have, respectively,

$$
\begin{aligned}
& \mathfrak{t}_{1}(-1) \overline{\mathfrak{t}}_{2}(-1)-\overline{\mathfrak{t}}_{1}(-1) \mathfrak{t}_{2}(-1) \\
& =\frac{\rho_{1}\left(\bar{\lambda}_{0}-\lambda_{0}\right)\left|\mathfrak{t}_{2}(-1)\right|^{2}}{\left|\alpha_{1}+\lambda_{0} \sin \theta_{1}\right|^{2}}, \\
& \mathfrak{u}_{1}(1) \overline{\mathfrak{t}}_{2}(1)-\overline{\mathfrak{t}}_{1}(1) \mathfrak{u}_{2}(1) \\
& =-\frac{\rho_{2}\left(\bar{\lambda}_{0}-\lambda_{0}\right)\left|\mathfrak{t}_{2}(1)\right|^{2}}{\left|\beta_{1}+\lambda_{0} \sin \theta_{2}\right|^{2}}, \\
& \mathfrak{t}_{1}(-0) \bar{u}_{2}(-0)-\overline{\mathfrak{t}}_{1}(-0) \mathfrak{t}_{2}(-0) \\
& =\delta^{2}\left[\mathfrak{t}_{1}(+0) \bar{u}_{2}(+0)-\overline{\mathfrak{t}}_{1}(+0) \mathfrak{t}_{2}(+0)\right] .
\end{aligned}
$$

Since $\lambda_{0} \neq \bar{\lambda}_{0}$, it follows from the last three equations and (19) that

$$
\begin{aligned}
\int_{-1}^{0}\left(\left|\mathfrak{t}_{1}(x)\right|^{2}+\left|\mathfrak{t}_{2}(x)\right|^{2}\right) d x & \\
& +\delta^{2} \int_{0}^{1}\left(\left|\mathfrak{t}_{1}(x)\right|^{2}+\left|\mathfrak{t}_{2}(x)\right|^{2}\right) d x \\
= & -\frac{\rho_{1}\left|\mathfrak{t}_{2}(-1)\right|^{2}}{\left|\alpha_{1}+\lambda_{0} \sin \theta_{1}\right|^{2}}-\frac{\rho_{2} \delta^{2}\left|\mathfrak{t}_{2}(1)\right|^{2}}{\left|\beta_{1}+\lambda_{0} \sin \theta_{2}\right|^{2}} .
\end{aligned}
$$

This contradicts the conditions $\int_{-1}^{0}\left(\left|\mathfrak{u}_{1}(x)\right|^{2}+\left|\mathfrak{u}_{2}(x)\right|^{2}\right) d x+$ $\delta^{2} \int_{0}^{1}\left(\left|\mathfrak{t}_{1}(x)\right|^{2}+\left|\mathfrak{t}_{2}(x)\right|^{2}\right) d x>0$ and $\rho_{i}>0, i=1,2$. Consequently, $\lambda_{0}$ must be real.

Now, we shall construct a special fundamental system of solutions of (12) for $\lambda$ not being an eigenvalue. Let us consider the next initial value problem:

$$
\begin{aligned}
\mathfrak{u}_{2}^{\prime}(x)-p_{1}(x) \mathfrak{u}_{1}(x) & =\lambda \mathfrak{u}_{1}(x), \\
\mathfrak{u}_{1}^{\prime}(x)+p_{2}(x) \mathfrak{u}_{2}(x) & =-\lambda \mathfrak{u}_{2}(x), \\
x & \in(-1,0), \\
\mathfrak{u}_{1}(-1)=\alpha_{2}+\lambda \cos \theta_{1}, \quad \mathfrak{u}_{2}(-1) & =\alpha_{1}+\lambda \sin \theta_{1} .
\end{aligned}
$$

By virtue of Theorem 1.1 in [31] this problem has a unique solution $\mathfrak{t}=\left(\begin{array}{l}\varphi_{11}(x, \lambda) \\ \varphi_{21}(x, \lambda)\end{array}\right)$, which is an entire function of $\lambda \in \mathbb{C}$ for each fixed $x \in[-1,0]$. Similarly, employing the same 
method as in proof of Theorem 1.1 in [31], we see that the problem

$$
\begin{gathered}
\mathfrak{t}_{2}^{\prime}(x)-p_{1}(x) \mathfrak{t}_{1}(x)=\lambda \mathfrak{t}_{1}(x), \\
\mathfrak{u}_{1}^{\prime}(x)+p_{2}(x) \mathfrak{t}_{2}(x)=-\lambda \mathfrak{t}_{2}(x), \\
x \in(0,1), \\
\mathfrak{t}_{1}(1)=\beta_{2}+\lambda \cos \theta_{2}, \quad \mathfrak{t}_{2}(1)=\beta_{1}+\lambda \sin \theta_{2},
\end{gathered}
$$

has a unique solution $\mathfrak{t}=\left(\begin{array}{l}\psi_{12}(x, \lambda) \\ \psi_{22}(x, \lambda)\end{array}\right)$ which is an entire function of parameter $\lambda$ for each fixed $x \in[0,1]$.

Now the functions $\varphi_{i 2}(x, \lambda)$ and $\psi_{i 1}(x, \lambda)$ are defined in terms of $\varphi_{i 1}(x, \lambda)$ and $\psi_{i 2}(x, \lambda), i=1,2$, respectively, as follows: the initial-value problem,

$$
\begin{aligned}
\mathfrak{t}_{2}^{\prime}(x)-p_{1}(x) \mathfrak{t}_{1}(x) & =\lambda \mathfrak{t}_{1}(x), \\
\mathfrak{t}_{1}^{\prime}(x)+p_{2}(x) \mathfrak{t}_{2}(x) & =-\lambda \mathfrak{t}_{2}(x), \\
x & \in(0,1), \\
\mathfrak{u}_{1}(0)=\frac{1}{\delta} \varphi_{11}(0, \lambda), & \mathfrak{t}_{2}(0)=\frac{1}{\delta} \varphi_{21}(0, \lambda),
\end{aligned}
$$

has unique solution $\mathfrak{u}=\left(\begin{array}{l}\varphi_{12}(x, \lambda) \\ \varphi_{22}(x, \lambda)\end{array}\right)$ for each $\lambda \in \mathbb{C}$.

Similarly, the following problem also has a unique solution $\mathfrak{t}=\left(\begin{array}{l}\psi_{11}(x, \lambda) \\ \psi_{21}(x, \lambda)\end{array}\right)$ :

$$
\begin{aligned}
\mathfrak{t}_{2}^{\prime}(x)-p_{1}(x) \mathfrak{t}_{1}(x) & =\lambda \mathfrak{u}_{1}(x), \\
\mathfrak{t}_{1}^{\prime}(x)+p_{2}(x) \mathfrak{t}_{2}(x) & =-\lambda \mathfrak{u}_{2}(x), \\
x & \in(-1,0), \\
\mathfrak{t}_{1}(0)=\delta \psi_{12}(0, \lambda), \quad \mathfrak{u}_{2}(0) & =\delta \psi_{22}(0, \lambda) .
\end{aligned}
$$

Let us construct two basic solutions of (12) as

$$
\varphi(\cdot, \lambda)=\left(\begin{array}{c}
\varphi_{1}(\cdot, \lambda) \\
\varphi_{2}(\cdot, \lambda)
\end{array}\right), \quad \psi(\cdot, \lambda)=\left(\begin{array}{c}
\psi_{1}(\cdot, \lambda) \\
\psi_{2}(\cdot, \lambda)
\end{array}\right),
$$

where

$$
\begin{aligned}
& \varphi_{1}(x, \lambda)= \begin{cases}\varphi_{11}(x, \lambda), & x \in[-1,0), \\
\varphi_{12}(x, \lambda), & x \in(0,1],\end{cases} \\
& \varphi_{2}(x, \lambda)= \begin{cases}\varphi_{21}(x, \lambda), & x \in[-1,0), \\
\varphi_{22}(x, \lambda), & x \in(0,1],\end{cases} \\
& \psi_{1}(x, \lambda)= \begin{cases}\psi_{11}(x, \lambda), & x \in[-1,0), \\
\psi_{12}(x, \lambda), & x \in(0,1],\end{cases} \\
& \psi_{2}(x, \lambda)= \begin{cases}\psi_{21}(x, \lambda), & x \in[-1,0), \\
\psi_{22}(x, \lambda), & x \in(0,1] .\end{cases}
\end{aligned}
$$

By virtue of (26) and (28) these solutions satisfy both transmission conditions (15). These functions are entire in $\lambda$ for all $x \in[-1,0) \cup(0,1]$.
Let $\mathscr{W}(\varphi, \psi)(\cdot, \lambda)$ denote the Wronskian of $\varphi(\cdot, \lambda)$ and $\psi(\cdot, \lambda)$ defined in [32, page 194], that is,

$$
\mathscr{W}(\varphi, \psi)(\cdot, \lambda):=\left|\begin{array}{ll}
\varphi_{1}(\cdot, \lambda) & \varphi_{2}(\cdot, \lambda) \\
\psi_{1}(\cdot, \lambda) & \psi_{2}(\cdot, \lambda)
\end{array}\right|
$$

It is obvious that the Wronskians,

$$
\begin{aligned}
\Omega_{i}(\lambda):= & \mathscr{W}(\varphi, \psi)(x, \lambda) \\
= & \varphi_{1 i}(x, \lambda) \psi_{2 i}(x, \lambda) \\
& -\varphi_{2 i}(x, \lambda) \psi_{1 i}(x, \lambda), \quad x \in \Gamma_{i}, i=1,2,
\end{aligned}
$$

are independent on variable $x \in \Gamma_{i}(i=1,2)$ and $\varphi_{i}(x, \lambda)$ and $\psi_{i}(x, \lambda)$ are entire functions of the parameter $\lambda$ for each $x \in \Gamma_{i}(i=1,2)$. Taking into account (26) and (28), a short calculation gives

$$
\Omega_{1}(\lambda)=\delta^{2} \Omega_{2}(\lambda)
$$

for each $\lambda \in \mathbb{C}$.

Corollary 2. The zeros of the functions $\Omega_{1}(\lambda)$ and $\Omega_{2}(\lambda)$ coincide.

Then, we may introduce to the consideration the characteristic function $\Omega(\lambda)$ as

$$
\Omega(\lambda):=\Omega_{1}(\lambda)=\delta^{2} \Omega_{2}(\lambda)
$$

Note that all eigenvalues of problems (12)-(15) are just zeros of the function $\Omega(\lambda)$. Indeed, since the functions $\varphi_{1}(x, \lambda)$ and $\varphi_{2}(x, \lambda)$ satisfy the boundary condition (13) and both transmission conditions (15), to find the eigenvalues of the (12)-(15), we have to insert the functions $\varphi_{1}(x, \lambda)$ and $\varphi_{2}(x, \lambda)$ in the boundary condition (14) and find the roots of this equation. In the following lemma, we show that all eigenvalues of the problems (12)-(15) are simple.

Lemma 3. The eigenvalues of the boundary value problems (12)-(15) form an at most countable set without finite limit points. All eigenvalues of the boundary value problems (12)(15) $($ of $\Omega(\lambda))$ are simple.

Proof. The eigenvalues are the zeros of the entire function occurring on the left-hand side in; see (35),

$$
\left(\beta_{1}+\lambda \sin \theta_{2}\right) \varphi_{12}(1, \lambda)-\left(\beta_{2}+\lambda \cos \theta_{2}\right) \varphi_{22}(1, \lambda)=0 .
$$

We have shown (see Lemma 1) that this function does not vanish for nonreal $\lambda$. In particular, it does not vanish identically. Therefore, its zeros form an at most countable set without finite limit points.

By (12) we obtain for $\lambda, \mu \in \mathbb{C}, \lambda \neq \mu$,

$$
\begin{aligned}
& \frac{d}{d x}\left\{\varphi_{1}(x, \lambda) \varphi_{2}(x, \mu)-\varphi_{1}(x, \mu) \varphi_{2}(x, \lambda)\right\} \\
& \quad=(\mu-\lambda)\left\{\varphi_{1}(x, \lambda) \varphi_{1}(x, \mu)+\varphi_{2}(x, \lambda) \varphi_{2}(x, \mu)\right\} .
\end{aligned}
$$


Integrating the above equation through $[-1,0]$ and $[0,1]$, and taking into account the initial conditions (23), (26), and (28), we obtain

$$
\begin{aligned}
& \delta^{2}\left(\varphi_{12}(1, \lambda) \varphi_{22}(1, \mu)-\varphi_{12}(1, \mu) \varphi_{22}(1, \lambda)\right) \\
& -(\mu-\lambda) \rho_{1} \\
& \quad=(\mu-\lambda)\left(\int _ { - 1 } ^ { 0 } \left(\varphi_{11}(x, \lambda) \varphi_{11}(x, \mu)\right.\right. \\
& \left.\quad+\varphi_{21}(x, \lambda) \varphi_{21}(x, \mu)\right) d x \\
& \quad+\delta^{2} \int_{0}^{1}\left(\varphi_{12}(x, \lambda) \varphi_{12}(x, \mu)\right. \\
& \left.\left.\quad+\varphi_{22}(x, \lambda) \varphi_{22}(x, \mu)\right) d x\right) .
\end{aligned}
$$

Dividing both sides of (38) by $(\lambda-\mu)$ and by letting $\mu \rightarrow \lambda$, we arrive to the relation

$$
\begin{gathered}
\delta^{2}\left(\varphi_{22}(1, \lambda) \frac{\partial \varphi_{12}(1, \lambda)}{\partial \lambda}-\varphi_{12}(1, \lambda) \frac{\partial \varphi_{22}(1, \lambda)}{\partial \lambda}\right)+\rho_{1} \\
=-\left(\int_{-1}^{0}\left(\left|\varphi_{11}(x, \lambda)\right|^{2}+\left|\varphi_{21}(x, \lambda)\right|^{2}\right) d x\right. \\
\left.+\delta^{2} \int_{0}^{1}\left(\left|\varphi_{12}(x, \lambda)\right|^{2}+\left|\varphi_{22}(x, \lambda)\right|^{2}\right) d x\right) .
\end{gathered}
$$

We show that equation

$$
\begin{aligned}
\Omega(\lambda)= & \mathscr{W}(\varphi, \psi)(1, \lambda) \\
=\delta^{2}\left(\left(\beta_{1}+\lambda \sin \theta_{2}\right) \varphi_{12}(1, \lambda)\right. & \left.\quad\left(\beta_{2}+\lambda \cos \theta_{2}\right) \varphi_{22}(1, \lambda)\right)=0,
\end{aligned}
$$

has only simple roots. Assume the converse, that is, (40) has a double root $\lambda^{*}$. Then the following two equations hold:

$$
\begin{aligned}
& \left(\beta_{1}+\lambda^{*} \sin \theta_{2}\right) \varphi_{12}\left(1, \lambda^{*}\right)-\left(\beta_{2}+\lambda^{*} \cos \theta_{2}\right) \varphi_{22}\left(1, \lambda^{*}\right)=0 \\
& \sin \theta_{2} \varphi_{12}\left(1, \lambda^{*}\right)+\left(\beta_{1}+\lambda^{*} \sin \theta_{2}\right) \frac{\partial \varphi_{12}\left(1, \lambda^{*}\right)}{\partial \lambda} \\
& \quad-\cos \theta_{2} \varphi_{22}\left(1, \lambda^{*}\right)-\left(\beta_{2}+\lambda^{*} \cos \theta_{2}\right) \frac{\partial \varphi_{22}\left(1, \lambda^{*}\right)}{\partial \lambda}=0 .
\end{aligned}
$$

Since $\rho_{2} \neq 0$ and $\lambda^{*}$ is real, then $\left(\beta_{1}+\lambda^{*} \sin \theta_{2}\right)^{2}+$ $\left(\beta_{2}+\lambda^{*} \cos \theta_{2}\right)^{2} \neq 0$. Let $\beta_{1}+\lambda^{*} \sin \theta_{2} \neq 0$. From (41)

$$
\begin{aligned}
\varphi_{12}\left(1, \lambda^{*}\right)= & \frac{\left(\beta_{2}+\lambda^{*} \cos \theta_{2}\right)}{\left(\beta_{1}+\lambda^{*} \sin \theta_{2}\right)} \varphi_{22}\left(1, \lambda^{*}\right), \\
\frac{\partial \varphi_{12}\left(1, \lambda^{*}\right)}{\partial \lambda}= & \frac{\rho_{2} \varphi_{22}\left(1, \lambda^{*}\right)}{\left(\beta_{1}+\lambda^{*} \sin \theta_{2}\right)^{2}} \\
& +\frac{\left(\beta_{2}+\lambda^{*} \cos \theta_{2}\right)}{\left(\beta_{1}+\lambda^{*} \sin \theta_{2}\right)} \frac{\partial \varphi_{22}\left(1, \lambda^{*}\right)}{\partial \lambda} .
\end{aligned}
$$

Combining (42) and (39) with $\lambda=\lambda^{*}$, we obtain

$$
\begin{aligned}
& \frac{\rho_{2} \delta^{2}\left(\varphi_{22}\left(1, \lambda^{*}\right)\right)^{2}}{\left(\beta_{1}+\lambda^{*} \sin \theta_{2}\right)^{2}}+\rho_{1} \\
& \quad=-\left(\int_{-1}^{0}\left(\left|\varphi_{11}(x, \lambda)\right|^{2}+\left|\varphi_{21}(x, \lambda)\right|^{2}\right) d x\right. \\
& \left.\quad+\delta^{2} \int_{0}^{1}\left(\left|\varphi_{12}(x, \lambda)\right|^{2}+\left|\varphi_{22}(x, \lambda)\right|^{2}\right) d x\right),
\end{aligned}
$$

contradicting the assumption $\rho_{i}>0, i=1,2$. The other case, when $\beta_{2}+\lambda^{*} \cos \theta_{2} \neq 0$ can be treated similarly and the proof is complete.

Recall that boundary value problems (12)-(15) have denumerable set of real and simple eigenvalues; see the above section. From (23) and (26), the solution of (12),

$$
\begin{aligned}
& \varphi(\cdot, \lambda)=\left(\begin{array}{l}
\varphi_{1}(\cdot, \lambda) \\
\varphi_{2}(\cdot, \lambda)
\end{array}\right), \\
& \varphi_{i}(x, \lambda)= \begin{cases}\varphi_{i 1}(x, \lambda), & x \in[-1,0), \\
\varphi_{i 2}(x, \lambda), & x \in(0,1], \\
& i=1,2,\end{cases}
\end{aligned}
$$

satisfies the following initial conditions

$$
\begin{aligned}
& \left(\begin{array}{ll}
\varphi_{11}(-1, \lambda) & \varphi_{12}\left(0^{+}, \lambda\right) \\
\varphi_{21}(-1, \lambda) & \varphi_{22}\left(0^{+}, \lambda\right)
\end{array}\right) \\
& \quad=\left(\begin{array}{ll}
\alpha_{2}+\lambda \cos \theta_{1} & \delta^{-1} \varphi_{11}\left(0^{-}, \lambda\right) \\
\alpha_{1}+\lambda \sin \theta_{1} & \delta^{-1} \varphi_{21}\left(0^{-}, \lambda\right)
\end{array}\right) .
\end{aligned}
$$

Since $\varphi(\cdot, \lambda)$ satisfies (13), then the eigenvalues of the problems (12)-(15) are the zeros of the function

$$
\begin{aligned}
\Omega(\lambda)=\delta^{2}( & \left(\beta_{1}+\lambda \sin \theta_{2}\right) \varphi_{12}(1, \lambda) \\
& \left.-\left(\beta_{2}+\lambda \cos \theta_{2}\right) \varphi_{22}(1, \lambda)\right)=0 .
\end{aligned}
$$

We shall transform (12), (23), (26), and (30) into the integral equations, see [32],

$$
\begin{aligned}
\varphi_{11}(x, \lambda)= & \alpha_{2} \cos \lambda(x+1)-\alpha_{1} \sin \lambda(x+1) \\
& +\lambda \cos \left(\lambda(x+1)+\theta_{1}\right)-\mathfrak{\mathfrak { I }}_{-1,1} \varphi_{11}(x, \lambda) \\
& -\widetilde{\mathfrak{T}}_{-1,2} \varphi_{21}(x, \lambda), \\
\varphi_{21}(x, \lambda)= & \alpha_{1} \cos \lambda(x+1)+\alpha_{2} \sin \lambda(x+1) \\
& +\lambda \sin \left(\lambda(x+1)+\theta_{1}\right)+\widetilde{\mathfrak{T}}_{-1,1} \varphi_{11}(x, \lambda) \\
& -\mathfrak{T}_{-1,2} \varphi_{21}(x, \lambda), \\
\varphi_{12}(x, \lambda)= & \frac{1}{\delta} \varphi_{11}\left(0^{-}, \lambda\right) \cos (\lambda x)-\frac{1}{\delta} \varphi_{21}\left(0^{-}, \lambda\right) \sin (\lambda x) \\
& -\mathfrak{T}_{0,1} \varphi_{12}(x, \lambda)-\widetilde{\mathfrak{T}}_{0,2} \varphi_{22}(x, \lambda),
\end{aligned}
$$




$$
\begin{aligned}
\varphi_{22}(x, \lambda)= & \frac{1}{\delta} \varphi_{11}\left(0^{-}, \lambda\right) \sin (\lambda x)+\frac{1}{\delta} \varphi_{21}\left(0^{-}, \lambda\right) \cos (\lambda x) \\
& +\widetilde{\mathfrak{I}}_{0,1} \varphi_{12}(x, \lambda)-\mathfrak{\mathfrak { I }}_{0,2} \varphi_{22}(x, \lambda),
\end{aligned}
$$

where $\mathfrak{\mathfrak { I }}_{-1, i}, \widetilde{\mathfrak{T}}_{-1, i}, \mathfrak{\mathfrak { I }}_{0, i}$ and $\widetilde{\mathfrak{I}}_{0, i}, i=1,2$, are the Volterra integral operators defined by

$$
\begin{aligned}
& \mathfrak{T}_{-1, i} \varphi(x, \lambda):=\int_{-1}^{x} \sin \lambda(x-t) p_{i}(t) \varphi(t, \lambda) d t, \\
& \widetilde{\mathfrak{I}}_{-1, i} \varphi(x, \lambda):=\int_{-1}^{x} \cos \lambda(x-t) p_{i}(t) \varphi(t, \lambda) d t, \\
& \mathfrak{T}_{0, i} \varphi(x, \lambda):=\int_{0}^{x} \sin \lambda(x-t) p_{i}(t) \varphi(t, \lambda) d t, \\
& \widetilde{\mathfrak{T}}_{0, i} \varphi(x, \lambda):=\int_{0}^{x} \cos \lambda(x-t) p_{i}(t) \varphi(t, \lambda) d t .
\end{aligned}
$$

For convenience, we define the constants

$$
\begin{gathered}
c_{1}:=\max \left\{\left|\alpha_{1}\right|+\left|\alpha_{2}\right|,\left|\sin \theta_{1}\right|+\left|\cos \theta_{1}\right|\right\}, \\
c_{2}:=\int_{-1}^{0}\left[\left|p_{1}(t)\right|+\left|p_{2}(t)\right|\right] d t, \quad c_{3}:=c_{1} c_{2} \exp \left(c_{2}\right), \\
c_{4}:=\int_{0}^{1}\left[\left|p_{1}(t)\right|+\left|p_{2}(t)\right|\right] d t, \quad c_{5}:=c_{3}+\frac{2}{|\delta|}\left(c_{1}+c_{3}\right), \\
c_{6}:=\max \left\{\left|\alpha_{1}\right|+\left|\alpha_{2}\right|,\left|\sin \theta_{2}\right|+\left|\cos \theta_{2}\right|\right\} .
\end{gathered}
$$

Now we define $\mathfrak{y}_{-1, i}(\cdot, \lambda)$ and $\mathfrak{y}_{0, i}(\cdot, \lambda), i=1,2$, to be

$$
\begin{aligned}
\mathfrak{y}_{-1,1}(x, \lambda) & :=\mathfrak{I}_{-1,1} \varphi_{11}(x, \lambda)+\widetilde{\mathfrak{T}}_{-1,2} \varphi_{21}(x, \lambda), \\
\mathfrak{y}_{-1,2}(x, \lambda) & :=\widetilde{\mathfrak{I}}_{-1,1} \varphi_{11}(x, \lambda)-\mathfrak{\mathfrak { I }}_{-1,2} \varphi_{21}(x, \lambda), \\
\mathfrak{y}_{0,1}(x, \lambda) & :=\mathfrak{\mathfrak { I }}_{0,1} \varphi_{12}(x, \lambda)+\widetilde{\mathfrak{T}}_{0,2} \varphi_{22}(x, \lambda), \\
\mathfrak{y}_{0,2}(x, \lambda) & :=\widetilde{\mathfrak{T}}_{0,1} \varphi_{12}(x, \lambda)-\mathfrak{\mathfrak { T }}_{0,2} \varphi_{22}(x, \lambda) .
\end{aligned}
$$

Lemma 4. The functions $\mathfrak{y}_{-1,1}(x, \lambda)$ and $\mathfrak{y}_{-1,2}(x, \lambda)$ are entire in $\lambda$ for any fixed $x \in[-1,0)$ and satisfy the growth condition

$$
\begin{array}{r}
\left|\mathfrak{y}_{-1,1}(x, \lambda)\right|, \\
\left|\mathfrak{y}_{-1,2}(x, \lambda)\right| \leq 2 c_{3}(1+|\lambda|) e^{|\Im \lambda|(x+1)}, \\
\lambda \in \mathbb{C} .
\end{array}
$$

Proof. Since $\mathfrak{y}_{-1,1}(x, \lambda)=\mathfrak{\mathfrak { I }}_{-1,1} \varphi_{11}(x, \lambda)+\widetilde{\mathfrak{I}}_{-1,2} \varphi_{21}(x, \lambda)$, then from (47) and (48) we obtain

$$
\begin{aligned}
\mathfrak{y}_{-1,1} & (x, \lambda) \\
= & \alpha_{2} \mathfrak{\mathfrak { T }}_{-1,1} \cos \lambda(x+1)-\alpha_{1} \mathfrak{\mathfrak { I }}_{-1,1} \sin \lambda(x+1) \\
& +\lambda \mathfrak{\mathfrak { I }}_{-1,1} \cos \left(\lambda(x+1)+\theta_{1}\right)-\mathfrak{\mathfrak { I }}_{-1,1} \mathfrak{y}_{-1,1}(x, \lambda) \\
& +\alpha_{1} \widetilde{\mathfrak{T}}_{-1,2} \cos \lambda(x+1)+\alpha_{2} \widetilde{\mathfrak{T}}_{-1,2} \sin \lambda(x+1) \\
& +\lambda \widetilde{\mathfrak{I}}_{-1,2} \sin \left(\lambda(x+1)+\theta_{1}\right)+\widetilde{\mathfrak{T}}_{-1,2} \mathfrak{y}_{-1,2}(x, \lambda) .
\end{aligned}
$$

Using the inequalities $|\sin z| \leq e^{|\Im z|}$ and $|\cos z| \leq e^{|\Im z|}$ for $z \in \mathbb{C}$ leads for $\lambda \in \mathbb{C}$ to

$$
\begin{aligned}
& \left|\mathfrak{y}_{-1,1}(x, \lambda)\right| \\
& \leq\left|\alpha_{2}\right|\left|\mathfrak{I}_{-1,1} \cos \lambda(x+1)\right|+\left|\alpha_{1} \mathfrak{I}_{-1,1} \sin \lambda(x+1)\right| \\
& +|\lambda|\left|\mathfrak{I}_{-1,1} \cos \left(\lambda(x+1)+\theta_{1}\right)\right|+\left|\mathfrak{I}_{-1,1} \mathfrak{y}_{-1,1}(x, \lambda)\right| \\
& +\left|\alpha_{1}\right|\left|\widetilde{\mathfrak{T}}_{-1,2} \cos \lambda(x+1)\right|+\left|\alpha_{2}\right|\left|\widetilde{\mathfrak{T}}_{-1,2} \sin \lambda(x+1)\right| \\
& +|\lambda|\left|\widetilde{\mathfrak{T}}_{-1,2} \sin \left(\lambda(x+1)+\theta_{1}\right)\right|+\left|\widetilde{\mathfrak{I}}_{-1,2} \mathfrak{y}_{-1,2}(x, \lambda)\right| \\
& \leq 2 c_{1}(1+|\lambda|) e^{|\Im \lambda|(x+1)} \int_{-1}^{x}\left[\left|p_{1}(t)\right|+\left|p_{2}(t)\right|\right] d t \\
& +e^{|\Im \lambda|(x+1)} \int_{-1}^{x}\left[\left|p_{1}(t)\right|\left|\mathfrak{y}_{-1,1}(t, \lambda)\right|\right. \\
& \left.+\left|p_{2}(t)\right|\left|\mathfrak{y}_{-1,2}(t, \lambda)\right|\right] e^{-|\Im \lambda|(t+1)} d t \\
& \leq 2 c_{1} c_{2}(1+|\lambda|) e^{|\Im \lambda|(x+1)} \\
& +e^{|\Im \lambda|(x+1)} \int_{-1}^{x}\left[\left|p_{1}(t)\right|\left|\mathfrak{y}_{-1,1}(t, \lambda)\right|\right. \\
& \left.+\left|p_{2}(t)\right|\left|\mathfrak{y}_{-1,2}(t, \lambda)\right|\right] e^{-|\Im \lambda|(t+1)} d t .
\end{aligned}
$$

The above inequality can be reduced to

$$
\begin{aligned}
& e^{-|\Im \lambda|(x+1)}\left|\mathfrak{y}_{-1,1}(x, \lambda)\right| \\
& \leq 2 c_{1} c_{2}(1+|\lambda|) \\
& \quad+\int_{-1}^{x}\left[\left|p_{1}(t)\right|\left|\mathfrak{y}_{-1,1}(t, \lambda)\right|\right. \\
& \left.\quad+\left|p_{2}(t)\right|\left|\mathfrak{y}_{-1,2}(t, \lambda)\right|\right] e^{-|\Im \lambda|(t+1)} d t
\end{aligned}
$$

Similarly, we can prove that

$$
\begin{aligned}
e^{-|\Im \lambda|(x+1)}\left|\mathfrak{y}_{-1,2}(x, \lambda)\right| \\
\leq 2 c_{1} c_{2}(1+|\lambda|) \\
\quad+\int_{-1}^{x}\left[\left|p_{1}(t)\right|\left|\mathfrak{y}_{-1,1}(t, \lambda)\right|\right. \\
\left.\quad+\left|p_{2}(t)\right|\left|\mathfrak{y}_{-1,2}(t, \lambda)\right|\right] e^{-|\Im \lambda|(t+1)} d t .
\end{aligned}
$$

Then from (57), (58), and Lemma 3.1 of [32, pp. 204], we obtain (54).

In a similar manner, we will prove the following lemma for $\mathfrak{y}_{0,1}(\cdot, \lambda)$ and $\mathfrak{y}_{0,2}(\cdot, \lambda)$.

Lemma 5. The functions $\mathfrak{y}_{0,1}(x, \lambda)$ and $\mathfrak{y}_{0,2}(x, \lambda)$ are entire in $\lambda$ for any fixed $x \in(0,1]$ and satisfy the growth condition

$$
\begin{aligned}
&\left|\mathfrak{y}_{0,1}(x, \lambda)\right|, \\
&\left|\mathfrak{y}_{0,2}(x, \lambda)\right| \leq 2 c_{4} \mathcal{c}_{5}(1+|\lambda|) e^{|\Im \lambda|(x+1)}, \\
& \lambda \in \mathbb{C} .
\end{aligned}
$$


Proof. Since $\mathfrak{y}_{0,1}(x, \lambda)=\mathfrak{\mathfrak { I }}_{0,1} \varphi_{12}(x, \lambda)+\widetilde{\mathfrak{I}}_{0,2} \varphi_{22}(x, \lambda)$, then from (49) and (50) we obtain

$$
\begin{aligned}
\mathfrak{y}_{0,1}(x, \lambda)= & \frac{1}{\delta} \varphi_{11}\left(0^{-}, \lambda\right) \mathfrak{I}_{0,1} \cos (\lambda x) \\
& -\frac{1}{\delta} \varphi_{21}\left(0^{-}, \lambda\right) \mathfrak{I}_{0,1} \sin (\lambda x) \\
& -\mathfrak{I}_{0,1} \mathfrak{y}_{-1,2}(x, \lambda)+\frac{1}{\delta} \varphi_{11}\left(0^{-}, \lambda\right) \widetilde{\mathfrak{I}}_{0,2} \sin (\lambda x) \\
& +\frac{1}{\delta} \varphi_{21}\left(0^{-}, \lambda\right) \widetilde{\mathfrak{I}}_{0,2} \cos (\lambda x)+\widetilde{\mathfrak{I}}_{0,2} \mathfrak{y}_{-1,2}(x, \lambda) .
\end{aligned}
$$

Then from (47) and (48) and Lemma 4, we get

$$
\begin{aligned}
\left|\mathfrak{y}_{0,1}(x, \lambda)\right| \leq & \frac{1}{|\delta|}\left|\varphi_{11}\left(0^{-}, \lambda\right)\right|\left|\mathfrak{I}_{0,1} \cos (\lambda x)\right| \\
& +\frac{1}{|\delta|}\left|\varphi_{21}\left(0^{-}, \lambda\right)\right|\left|\mathfrak{I}_{0,1} \sin (\lambda x)\right| \\
& +\left|\mathfrak{I}_{0,1} \mathfrak{y}_{-1,2}(x, \lambda)\right| \\
& +\frac{1}{|\delta|}\left|\varphi_{11}\left(0^{-}, \lambda\right)\right|\left|\widetilde{\mathfrak{I}}_{0,2} \sin (\lambda x)\right| \\
& +\frac{1}{|\delta|}\left|\varphi_{21}\left(0^{-}, \lambda\right)\right|\left|\widetilde{\mathfrak{I}}_{0,2} \cos (\lambda x)\right| \\
& +\left|\widetilde{\mathfrak{I}}_{0,2} \mathfrak{y}_{-1,2}(x, \lambda)\right| \\
\leq & 2 c_{4}(1+|\lambda|) \\
& \times\left(c_{3}+\frac{2}{|\delta|}\left(c_{1}+c_{3}\right)\right) e^{|\Im \lambda|(x+1)} \\
= & 2 c_{4} c_{5}(1+|\lambda|) e^{|\mathfrak{\Im} \lambda|(x+1)} .
\end{aligned}
$$

Similarly, we can prove that

$$
\left|\mathfrak{y}_{0,2}(x, \lambda)\right| \leq 2 c_{4} \mathcal{c}_{5}(1+|\lambda|) e^{|\Im \lambda|(x+1)} .
$$

\section{The Numerical Scheme}

In this section we derive the method of computing eigenvalues of problems (12)-(15) numerically. The basic idea of the scheme is to split $\Omega(\lambda)$ into two parts a known part $\mathscr{K}(\lambda)$ and an unknown one $\mathcal{U}(\lambda)$. Then we approximate $\mathscr{U}(\lambda)$ using (5) to get the approximate $\Omega(\lambda)$ and then compute the approximate zeros. We first split $\Omega(\lambda)$ into two parts:

$$
\Omega(\lambda):=\mathscr{K}(\lambda)+\mathscr{U}(\lambda),
$$

where $\mathcal{U}(\lambda)$ is the unknown part involving integral operators

$$
\begin{aligned}
\mathcal{U}(\lambda):=\delta[ & \left(\beta_{2}+\lambda \cos \theta_{2}\right) \sin \lambda \\
& \left.-\left(\beta_{1}+\lambda \sin \theta_{2}\right) \cos \lambda\right] \mathfrak{y}_{-1,1}\left(0^{-}, \lambda\right) \\
-\delta[ & \left(\beta_{1}+\lambda \sin \theta_{2}\right) \sin \lambda \\
& \left.+\left(\beta_{2}+\lambda \cos \theta_{2}\right) \cos \lambda\right] \mathfrak{y}_{-1,2}\left(0^{-}, \lambda\right)
\end{aligned}
$$

$$
\begin{aligned}
-\delta^{2}\left[\left(\beta_{1}+\lambda \sin \theta_{2}\right) \mathfrak{y}_{0,1}(1, \lambda)\right. & \\
& \left.+\left(\beta_{2}+\lambda \cos \theta_{2}\right) \mathfrak{y}_{0,2}(1, \lambda)\right],
\end{aligned}
$$

and $\mathscr{K}(\lambda)$ is the known part

$$
\begin{aligned}
\mathscr{K}(\lambda):= & \delta\left(\beta_{1}+\lambda \sin \theta_{2}\right) \\
& \times\left[\alpha_{2} \cos 2 \lambda-\alpha_{1} \sin 2 \lambda+\lambda \cos \left(2 \lambda+\theta_{1}\right)\right] \\
& -\delta\left(\beta_{2}+\lambda \cos \theta_{2}\right) \\
& \times\left[\alpha_{2} \sin 2 \lambda+\alpha_{1} \cos 2 \lambda+\lambda \sin \left(2 \lambda+\theta_{1}\right)\right] .
\end{aligned}
$$

Then, from Lemmas 4 and 5, we have the following result.

Lemma 6. The function $\mathcal{U}(\lambda)$ is entire in $\lambda$ and the following estimate holds:

$$
|\mathcal{U}(\lambda)| \leq \phi(\lambda) e^{2|\Im \lambda|}
$$

where

$$
\begin{gathered}
\phi(\lambda):=M(1+|\lambda|)^{2}, \\
M:=4|\delta| c_{6}\left(2 c_{3}+|\delta| c_{4} c_{5}\right) .
\end{gathered}
$$

Proof. From (64), we have

$$
\begin{aligned}
|\mathcal{U}(\lambda)| \leq & |\delta|\left[\left(\left|\beta_{2}\right|+|\lambda|\left|\cos \theta_{2}\right|\right)|\sin \lambda|\right. \\
& \left.+\left(\left|\beta_{1}\right|+|\lambda|\left|\sin \theta_{2}\right|\right)|\cos \lambda|\right]\left|\mathfrak{y}_{-1,1}\left(0^{-}, \lambda\right)\right| \\
+ & +|\delta|\left[\left(\left|\beta_{1}\right|+|\lambda|\left|\sin \theta_{2}\right|\right)|\sin \lambda|\right. \\
& \left.\quad+\left(\left|\beta_{2}\right|+\left|\lambda \cos \theta_{2}\right|\right)|\cos \lambda|\right]\left|\mathfrak{y}_{-1,2}\left(0^{-}, \lambda\right)\right| \\
+ & \delta^{2}\left[\left(\left|\beta_{1}\right|+|\lambda|\left|\sin \theta_{2}\right|\right)\left|\mathfrak{y}_{0,1}(1, \lambda)\right|\right. \\
& \left.+\left(\left|\beta_{2}\right|+|\lambda|\left|\cos \theta_{2}\right|\right)\left|\mathfrak{y}_{0,2}(1, \lambda)\right|\right] .
\end{aligned}
$$

Using the inequalities $|\sin \lambda| \leq e^{|\Im \lambda|}$ and $|\cos \lambda| \leq e^{|\Im \lambda|}$ for $\lambda \in \mathbb{C}$, and Lemmas 4 and 5 imply (66).

Thus $\mathcal{U}(\lambda)$ is an entire function of exponential type $\sigma=2$. In the following we let $\lambda \in \mathbb{R}$ since all eigenvalues are real. Now we approximate the function $\mathscr{U}(\lambda)$ using the operator (5) where $h \in(0, \pi / 2)$ and $\omega:=(\pi-2 h) / 2$ and then, from (7), we obtain

$$
\left|\mathcal{U}(\lambda)-\left(\mathscr{G}_{h, N} \mathscr{U}\right)(\lambda)\right| \leq T_{h, N}(\lambda),
$$

where

$$
\begin{aligned}
T_{h, N}(\lambda):= & 2\left|\sin \left(h^{-1} \pi \lambda\right)\right| \\
& \times \phi(|\Re \lambda|+h(N+1)) \frac{e^{-\omega N}}{\sqrt{\pi \omega N}} \beta_{N}(0), \quad \lambda \in \mathbb{R} .
\end{aligned}
$$

The samples, $\mathscr{U}(n h)=\Omega(n h)-\mathscr{K}(n h), n \in \mathbb{Z}_{N}(\lambda)$, cannot be computed explicitly in the general case. We approximate these 
samples numerically by solving the initial-value problems defined by (12) and (45) to obtain the approximate values $\widetilde{\mathscr{U}}(n h), n \in \mathbb{Z}_{N}(\lambda)$, that is, $\widetilde{\mathscr{U}}(n h)=\widetilde{\Omega}(n h)-\mathscr{K}(n h)$. Here we use a computer algebra system, MATHEMATICA, to obtain the approximate solutions with the required accuracy. However, a separate study for the effect of different numerical schemes and the computational costs would be interesting. Accordingly we have the explicit expansion

$$
\begin{aligned}
\left(\mathscr{G}_{h, N} \widetilde{\mathscr{U}}\right)(\lambda):= & \sum_{n \in \mathbb{Z}_{N}(\lambda)} \widetilde{\mathscr{U}}(n h) \operatorname{sinc}\left(h^{-1} \pi x-n \pi\right) \\
& \times G\left(\frac{\sqrt{\omega}(\lambda-n h)}{\sqrt{N} h}\right) .
\end{aligned}
$$

Therefore we get, cf. (10),

$$
\left|\left(\mathscr{G}_{h, N} \mathscr{U}\right)(\lambda)-\left(\mathscr{G}_{h, N} \widetilde{\mathscr{U}}\right)(\lambda)\right| \leq A_{\varepsilon, N}(0), \quad \lambda \in \mathbb{R} .
$$

Now let $\widetilde{\Omega}_{N}(\lambda):=\mathscr{K}(\lambda)+\left(\mathscr{G}_{h, N} \widetilde{\mathscr{U}}\right)(\lambda)$. From (69) and (72) we obtain

$$
\left|\Omega(\lambda)-\widetilde{\Omega}_{N}(\lambda)\right| \leq T_{h, N}(\lambda)+A_{\varepsilon, N}(0), \quad \lambda \in \mathbb{R} .
$$

Let $\lambda^{*}$ be an eigenvalue and $\lambda_{N}$ its desired approximation, that is, $\Omega\left(\lambda^{*}\right)=0$ and $\widetilde{\Omega}_{N}\left(\lambda_{N}\right)=0$. From (73) we have $\left|\widetilde{\Omega}_{N}\left(\lambda^{*}\right)\right| \leq T_{h, N}\left(\lambda^{*}\right)+A_{\varepsilon, N}(0)$. Define the curves

$$
a_{ \pm}(\lambda)=\widetilde{\Omega}_{N}(\lambda) \pm T_{h, N}(\lambda)+A_{\varepsilon, N}(0) .
$$

The curves $a_{+}(\lambda), a_{-}(\lambda)$ enclose the curve of $\Omega(\lambda)$ for suitably large $N$. Hence the closure interval is determined by solving $a_{ \pm}(\lambda)=0$, giving an interval

$$
I_{\varepsilon, N}:=\left[a_{-}, a_{+}\right] \text {. }
$$

It is worthwhile to mention that the simplicity of the eigenvalues guarantees the existence of approximate eigenvalues, that is, the $\lambda_{N}$ 's for which $\widetilde{\Omega}_{N}\left(\lambda_{N}\right)=0$. Next we estimate the error $\left|\lambda^{*}-\lambda_{N}\right|$ for the eigenvalue $\lambda^{*}$.

Theorem 7. Let $\lambda^{*}$ be an eigenvalue of (12)-(15) and let $\lambda_{N}$ be its approximation. Then, for $\lambda \in \mathbb{R}$, we have the following estimate:

$$
\left|\lambda^{*}-\lambda_{N}\right|<\frac{T_{h, N}\left(\lambda_{N}\right)+A_{\varepsilon, N}(0)}{\inf _{\zeta \in I_{\varepsilon, N}}\left|\Omega^{\prime}(\zeta)\right|},
$$

where the interval $I_{\varepsilon, N}$ is defined above.

Proof. Replacing $\lambda$ by $\lambda_{N}$ in (73) we obtain

$$
\left|\Omega\left(\lambda_{N}\right)-\Omega\left(\lambda^{*}\right)\right|<T_{h, N}\left(\lambda_{N}\right)+A_{\varepsilon, N}(0),
$$

where we have used $\widetilde{\Omega}_{N}\left(\lambda_{N}\right)=\Omega\left(\lambda^{*}\right)=0$. Using the mean value theorem yields that for some $\zeta \in J_{\varepsilon, N}:=$ $\left[\min \left(\lambda^{*}, \lambda_{N}\right), \max \left(\lambda^{*}, \lambda_{N}\right)\right]$,

$$
\left|\left(\lambda^{*}-\lambda_{N}\right) \Omega^{\prime}(\zeta)\right| \leq T_{h, N}\left(\lambda_{N}\right)+A_{\varepsilon, N}(0), \quad \zeta \in J_{\varepsilon, N} \subset I_{\varepsilon, N} .
$$

Since $\lambda^{*}$ is simple and $N$ is sufficiently large, then $\inf _{\zeta \in I_{\varepsilon, N}}\left|\Omega^{\prime}(\zeta)\right|>0$ and we get (76).

\section{Numerical Examples}

In this section, we introduce two examples illustrating the the above method. Also, in the following examples, we observe that the exact solutions $\lambda_{k}$ are all inside the interval $\left[a_{-}, a_{+}\right]$. In these two examples, we indicate the effect of the amplitude error in the method by determining enclosure intervals for different values of $\varepsilon$. We also indicate the effect of $N$ and $h$ by several choices. The eigenvalues of the following examples cannot computed concretely, then we use MATHEMATICA to obtain the exact values. MATHEMATICA is also used in rounding off the exact eigenvalues, which are square roots. Both numerical results and the associated figures prove the credibility of the method.

Example 1. Consider the system

$$
\begin{gathered}
y_{2}^{\prime}(x)-p(x) y_{1}(x)=\lambda y_{1}(x), \\
y_{1}^{\prime}(x)+p(x) y_{2}(x)=-\lambda y_{2}(x), \\
x \in[-1,0) \cup(0,1], \\
\lambda y_{1}(-1)-(\sqrt{2}+\lambda) y_{2}(-1)=0, \\
(\sqrt{2}+\lambda) y_{1}(1)-\lambda y_{2}(1)=0, \\
y_{1}\left(0^{-}\right)-2 y_{1}\left(0^{+}\right)=0, \quad y_{2}\left(0^{-}\right)-2 y_{2}\left(0^{+}\right)=0 .
\end{gathered}
$$

Here

$$
p_{1}(x)=p_{2}(x)=r(x)= \begin{cases}x, & x \in[-1,0), \\ 1, & (0,1]\end{cases}
$$

$\theta_{1}=\theta_{2}=\pi / 4, \alpha_{1}=\beta_{2}=0, \alpha_{2}=\beta_{1}=1$, and $\delta=2$. Direct calculations give

$$
\begin{gathered}
\mathscr{K}(\lambda)=2((\sqrt{2} \lambda+1) \cos (2 \lambda)-\lambda(\lambda+\sqrt{2}) \sin (2 \lambda)), \\
\Omega(\lambda)=2\left((\sqrt{2} \lambda+1) \cos \left(2 \lambda+\frac{1}{2}\right)\right. \\
\left.-\lambda(\lambda+\sqrt{2}) \sin \left(2 \lambda+\frac{1}{2}\right)\right) .
\end{gathered}
$$

As is clearly seen, the eigenvalues cannot be computed explicitly. Tables 1, 2, and 3 indicate the application of our technique to this problem and the effect of $\varepsilon$. By exact we mean the zeros of $\Delta(\lambda)$ computed by Mathematica.

Figures 1 and 2 illustrate the enclosure intervals dominating $\lambda_{0}$ for $N=25, h=0.1$ and $\varepsilon=10^{-2}, \varepsilon=10^{-5}$, respectively. The middle curve represents $\Delta(\lambda)$, while the upper and lower curves represent the curves of $a_{+}(\lambda), a_{-}(\lambda)$, respectively. We notice that when $\varepsilon=10^{-5}$, the two curves are almost identical. Similarly, Figures 3 and 4 illustrate the enclosure intervals dominating $\lambda_{1}$ for $h=0.1, N=25$ and $\varepsilon=10^{-2}, \varepsilon=10^{-5}$, respectively. 
TABLE 1: The approximation $\lambda_{k, N}$ and the exact solutions $\lambda_{k}$ for different choices of $h$ and $N$.

\begin{tabular}{|c|c|c|c|c|c|c|}
\hline & $\lambda_{k}$ & & $\lambda_{-2}$ & $\lambda_{-1}$ & $\lambda_{0}$ & $\lambda_{1}$ \\
\hline \multirow{2}{*}{$\lambda_{k, N}$} & $\begin{array}{l}\quad \text { Exact } \lambda_{k} \\
h=0.6 \\
\omega=0.9708\end{array}$ & $\begin{array}{l}N=15 \\
N=25\end{array}$ & $\begin{array}{l}-1.2061856254879546 \\
-1.2061855965725146 \\
-1.2061856254843495\end{array}$ & $\begin{array}{l}-0.5151195632138896 \\
-0.5151190347168934 \\
-0.5151195631493376\end{array}$ & $\begin{array}{c}0.34274095892547224 \\
0.34274053593937165 \\
0.342740958869788\end{array}$ & $\begin{array}{l}1.616237903407542 \\
1.616237692038969 \\
1.6162379033820231\end{array}$ \\
\hline & $\begin{array}{c}h=0.1 \\
\omega=1.4708\end{array}$ & $\begin{array}{l}N=15 \\
N=25\end{array}$ & $\begin{array}{l}-1.2061856254844203 \\
-1.2061856254879548 \\
\end{array}$ & $\begin{array}{l}-0.5151195632158179 \\
-0.5151195632138894 \\
\end{array}$ & $\begin{array}{c}0.3427409589350932 \\
0.34274095892547236\end{array}$ & $\begin{array}{l}1.6162379034134882 \\
1.6162379034075425\end{array}$ \\
\hline
\end{tabular}

TABLE 2: Absolute error $\left|\lambda_{k}-\lambda_{k, N}\right|$.

\begin{tabular}{|c|c|c|c|c|c|}
\hline \multicolumn{2}{|c|}{$\lambda_{k}$} & $\lambda_{-2}$ & $\lambda_{-1}$ & $\lambda_{0}$ & $\lambda_{1}$ \\
\hline \multirow{2}{*}{$h=0.6$} & $N=15$ & $2.89154 \times 10^{-8}$ & $5.28497 \times 10^{-7}$ & $4.22986 \times 10^{-7}$ & $2.11369 \times 10^{-7}$ \\
\hline & $N=25$ & $3.60512 \times 10^{-12}$ & $6.4552 \times 10^{-11}$ & $5.56842 \times 10^{-11}$ & $2.55189 \times 10^{-11}$ \\
\hline \multirow{2}{*}{$h=0.1$} & $N=15$ & $3.53428 \times 10^{-12}$ & $1.92824 \times 10^{-12}$ & $9.62097 \times 10^{-12}$ & $5.94613 \times 10^{-12}$ \\
\hline & $N=25$ & $2.22045 \times 10^{-16}$ & $2.22045 \times 10^{-16}$ & $5.55112 \times 10^{-17}$ & $4.44089 \times 10^{-16}$ \\
\hline
\end{tabular}

TABLE 3: For $N=25$ and $h=0.1$, the exact solutions $\lambda_{k}$ are all inside the interval $\left[a_{-}, a_{+}\right]$for different values of $\varepsilon$.

\begin{tabular}{lcccc}
\hline$\lambda_{k}$ & $\lambda_{-2}$ & $\lambda_{-1}$ & $\lambda_{0}$ & $\lambda_{1}$ \\
\hline Exact $\lambda_{k}$ & -1.2061856254879546 & -0.5151195632138896 & 0.34274095892547224 & 1.616237903407542 \\
$I_{\varepsilon, N}, \varepsilon=10^{-2}$ & {$[-1.2335696,-1.1771922]$} & {$[-0.5486790,-0.4826816]$} & {$[0.3246478,0.3601346]$} & {$[1.6100119,1.6224066]$} \\
$I_{\varepsilon, N}, \varepsilon=10^{-5}$ & {$[-1.2062137,-1.2061575]$} & {$[-0.5151525,-0.5150867]$} & {$[0.3427232,0.3427586]$} & {$[1.6162317,1.6162441]$} \\
\hline
\end{tabular}

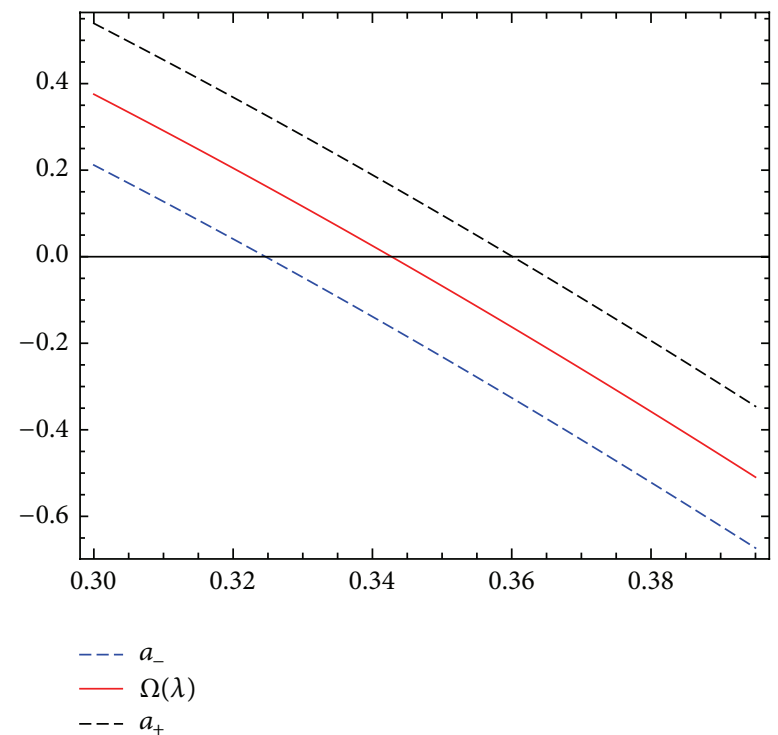

FIgURE 1: The enclosure interval dominating $\lambda_{0}$ for $h=0.1, N=25$, and $\varepsilon=10^{-2}$.

Example 2. In this example we consider the system

$$
\begin{gathered}
y_{2}^{\prime}(x)-p(x) y_{1}(x)=\lambda y_{1}(x), \\
y_{1}^{\prime}(x)+p(x) y_{2}(x)=-\lambda y_{2}(x), \\
x \in[-1,0) \cup(0,1], \\
\sqrt{3} y_{1}(-1)-y_{2}(-1)=0, \\
\left(1+\frac{1}{2} \lambda\right) y_{1}(1)-\left(1+\frac{\sqrt{3}}{2} \lambda\right) y_{2}(1)=0, \\
y_{1}\left(0^{-}\right)-3 y_{1}\left(0^{+}\right)=0, \quad y_{2}\left(0^{-}\right)-3 y_{2}\left(0^{+}\right)=0,
\end{gathered}
$$

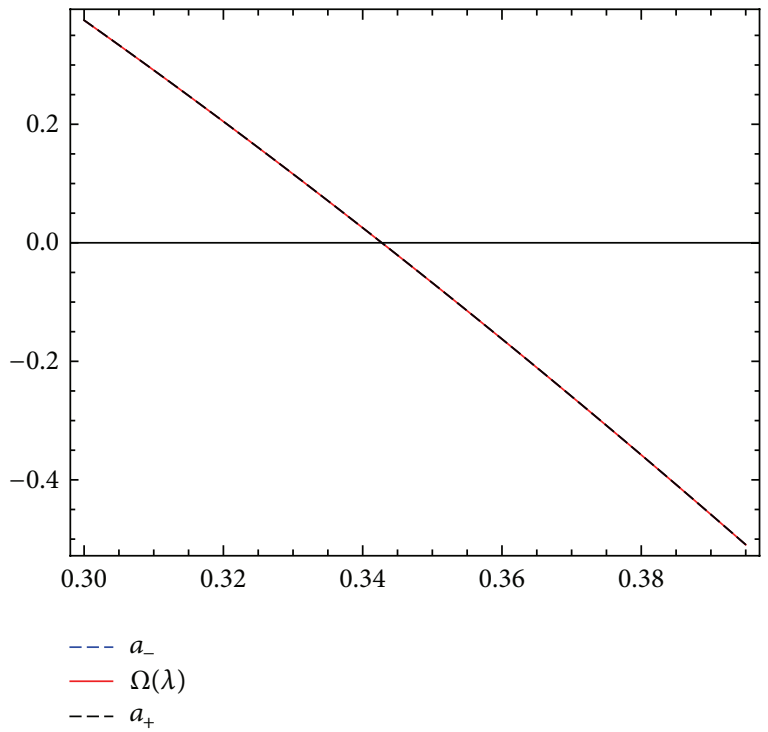

FIGURE 2: The enclosure interval dominating $\lambda_{0}$ for $h=0.1, N=25$, and $\varepsilon=10^{-5}$.

where

$$
p_{1}(x)=p_{2}(x)=p(x)= \begin{cases}x, & x \in[-1,0), \\ x^{2}, & (0,1],\end{cases}
$$

$\theta_{1}=\theta_{2}=0, \alpha_{1}=\beta_{2}=1, \alpha_{2}=\beta_{1}=2$, and $\delta=1 / 2$. Direct calculations give

$$
\begin{gathered}
\mathscr{K}(\lambda)=\frac{1}{2}\left((\lambda+3) \cos (2 \lambda)-\left(\lambda^{2}+3 \lambda+4\right) \sin (2 \lambda)\right), \\
\Omega(\lambda)=\frac{1}{2}\left(\left(\lambda^{2}+3 \lambda+4\right) \sin \left(\frac{1}{6}-2 \lambda\right)\right. \\
\left.+(\lambda+3) \cos \left(\frac{1}{6}-2 \lambda\right)\right) .
\end{gathered}
$$


TABLE 4: The approximation $\lambda_{k, N}$ and the exact solutions $\lambda_{k}$ for different choices of $h$ and $N$.

\begin{tabular}{|c|c|c|c|c|c|c|}
\hline & $\lambda_{k}$ & & $\lambda_{-2}$ & $\lambda_{-1}$ & $\lambda_{0}$ & $\lambda_{1}$ \\
\hline \multirow{5}{*}{$\lambda_{k, N}$} & Exact $\lambda_{k}$ & & -3.0661256186938894 & -1.0959703083466883 & 0.3689030343814017 & 1.8338437675275228 \\
\hline & $h=0.8$ & $N=15$ & -3.066123822200742 & -1.0959640944020903 & 0.36890463046491767 & 1.8338437607680411 \\
\hline & $\omega=0.0 .7708$ & $N=25$ & -3.0661256172349627 & -1.0959703026494807 & 0.36890303588091056 & 1.8338437676561417 \\
\hline & $h=0.2$ & $N=15$ & -3.0661256186844446 & -1.0959703083278305 & 0.3689030343916001 & 1.833843767517251 \\
\hline & $\omega=1.3708$ & $N=25$ & -3.06612561869389 & -1.095970308346688 & 0.3689030343814024 & 1.8338437675275225 \\
\hline
\end{tabular}

TABLE 5: Absolute error $\left|\lambda_{k}-\lambda_{k, N}\right|$.

\begin{tabular}{|c|c|c|c|c|c|}
\hline \multicolumn{2}{|c|}{$\lambda_{k}$} & \multirow{2}{*}{$\frac{\lambda_{-2}}{1.79649 \times 10^{-6}}$} & \multirow{2}{*}{$\frac{\lambda_{-1}}{6.21394 \times 10^{-6}}$} & \multirow{2}{*}{$\frac{\lambda_{0}}{1.59608 \times 10^{-6}}$} & \multirow{2}{*}{$\frac{\lambda_{1}}{6.75948 \times 10^{-9}}$} \\
\hline$h=0.8$ & $N=15$ & & & & \\
\hline$n=0.0$ & $N=25$ & $1.45893 \times 10^{-9}$ & $5.69721 \times 10^{-9}$ & $1.49951 \times 10^{-9}$ & $1.28619 \times 10^{-10}$ \\
\hline \multirow{2}{*}{$h=0.2$} & $N=15$ & $9.44489 \times 10^{-12}$ & $1.88578 \times 10^{-12}$ & $1.01984 \times 10^{-11}$ & $1.02718 \times 10^{-11}$ \\
\hline & $N=25$ & $4.44089 \times 10^{-16}$ & $2.22045 \times 10^{-16}$ & $6.66134 \times 10^{-16}$ & $2.22045 \times 10^{-16}$ \\
\hline
\end{tabular}

TABLE 6: For $N=25$ and $h=0.2$, the exact solutions $\lambda_{k}$ are all inside the interval $\left[a_{-}, a_{+}\right]$for different values of $\varepsilon$.

\begin{tabular}{lcccc}
\hline$\lambda_{k}$ & $\lambda_{-2}$ & $\lambda_{-1}$ & $\lambda_{0}$ & $\lambda_{1}$ \\
\hline Exact $\lambda_{k}$ & -3.0661256186938894 & -1.0959703083466883 & 0.3689030343814017 & 1.8338437675275228 \\
$I_{\varepsilon, N}, \varepsilon=10^{-2}$ & {$[-3.1098742,-3.0188665]$} & {$[-1.1631502,-1.0339754]$} & {$[0.3437434,0.3933486]$} & {$[1.8220764,1.8454828]$} \\
$I_{\varepsilon, N}, \varepsilon=10^{-5}$ & {$[-3.0661709,-3.0660803]$} & {$[-1.0960346,-1.0959060]$} & {$[0.3688783,0.3689278]$} & {$[1.8338321,1.8338555]$} \\
\hline
\end{tabular}

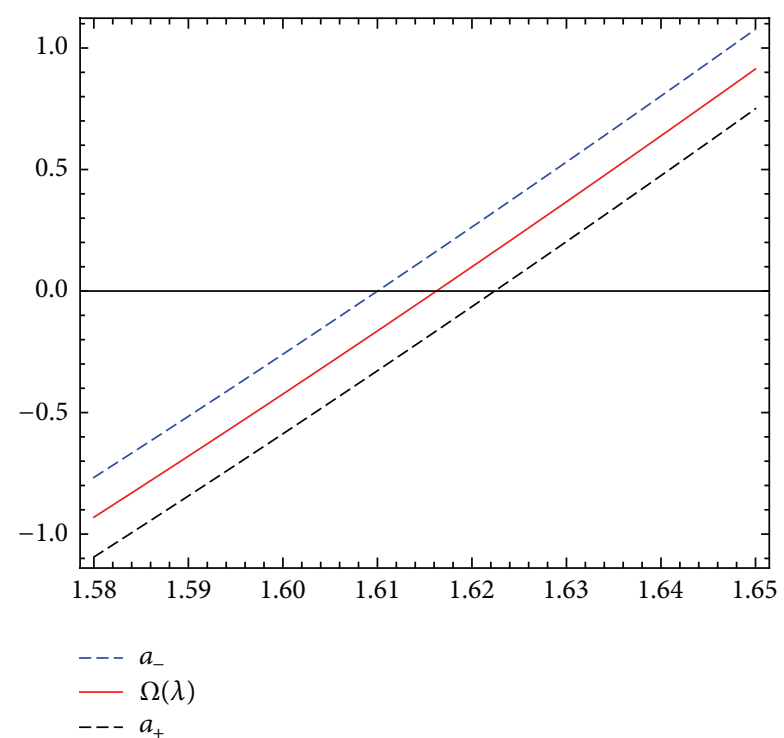

FIGURE 3: The enclosure interval dominating $\lambda_{1}$ for $h=0.1, N=25$, and $\varepsilon=10^{-2}$.

Tables 4 and 6 give the exact eigenvalues $\left\{\lambda_{k}\right\}_{k=-2}^{1}$ and their approximate ones for different values of $h, N$, and $\varepsilon$. In Table 5, we give the absolute error for different values of $h$ and N.

Here, Figures 5, 6, 7, and 8 illustrate the enclosure intervals dominating $\lambda_{-2}$ and $\lambda_{-1}$ for $h=0.2, N=25$ and $\varepsilon=10^{-2}, \varepsilon=10^{-5}$, respectively.

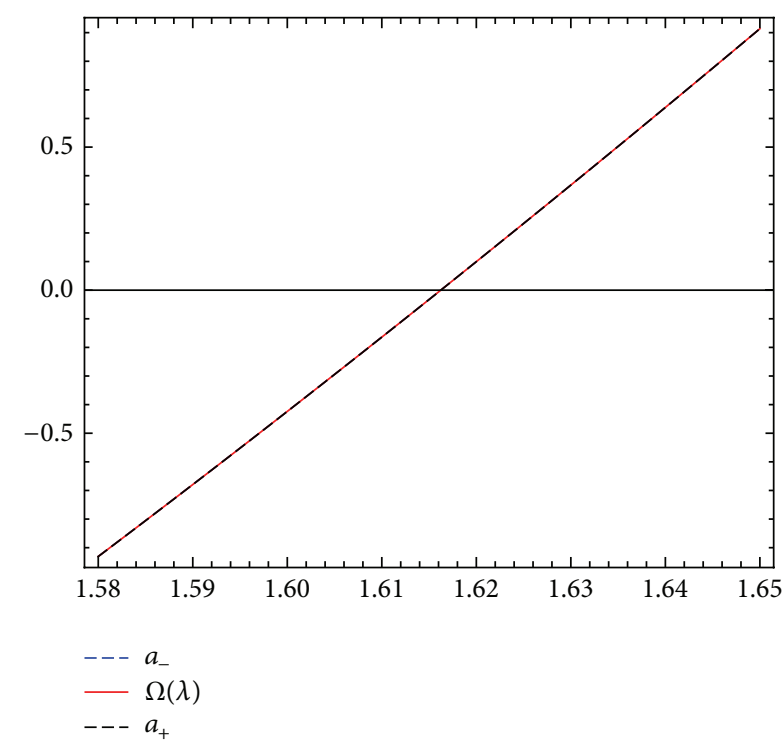

FIgURE 4: The enclosure interval dominating $\lambda_{1}$ for $h=0.1, N=25$, and $\varepsilon=10^{-5}$.

\section{Conclusion}

With a simple analysis and with values of solutions of initial value problems computed at a few values of the eigenparameter, we have computed the eigenvalues of a Dirac system which has a discontinuity at one point and contains a spectral parameter in all boundary conditions, with a certain estimated error. The method proposed is 


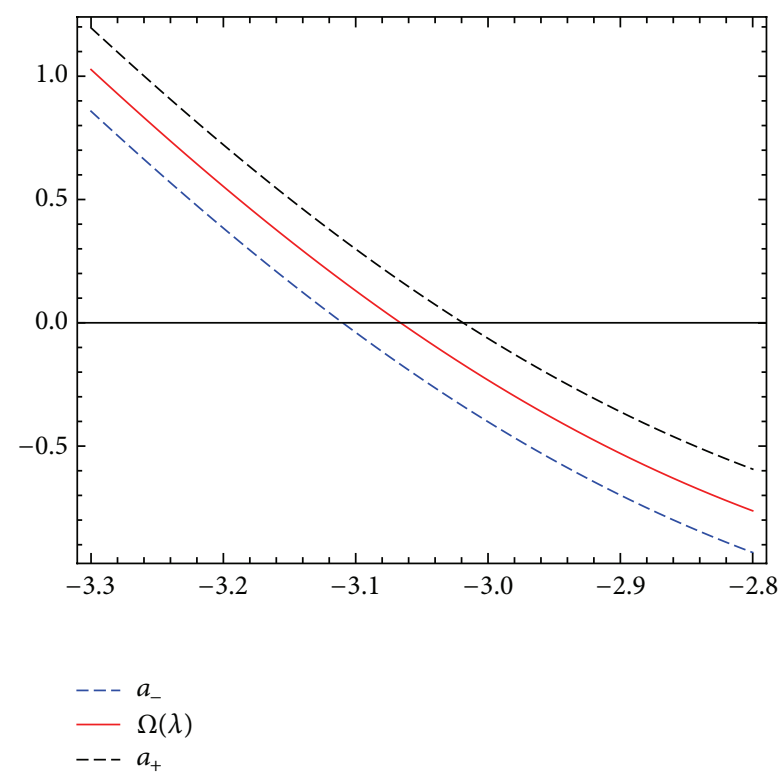

FIGURE 5: The enclosure interval dominating $\lambda_{-2}$ for $h=0.2, N=$ 25 , and $\varepsilon=10^{-2}$.

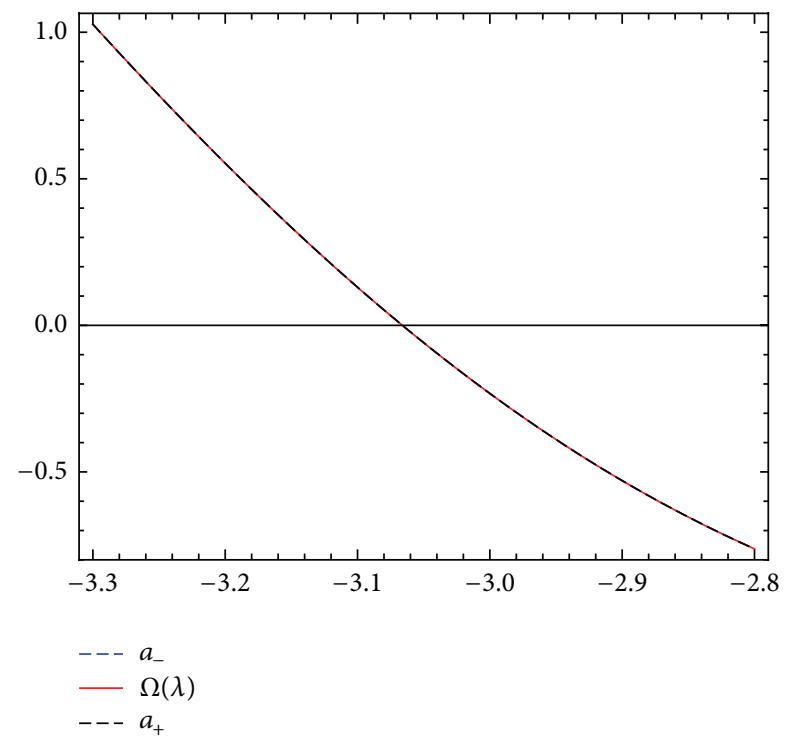

FIGURE 6: The enclosure interval dominating $\lambda_{-2}$ for $h=0.2, N=$ 25 , and $\varepsilon=10^{-5}$.

a shooting procedure; that is, the problem is reformulated as two initial value ones, due to the interior discontinuity, of size two and a miss-distance is defined at the right end of the interval of integration whose roots are the eigenvalues to be computed. The unknown part $\mathcal{U}(\lambda)$ of the miss-distance can be written in terms of a function which is an entire function of exponential type. Therefore, we propose to approximate such term by means of a truncated cardinal series with sampling values approximated by solving numerically corresponding suitable initial value problems. Finally, in Section 4 we introduced two instructive examples, where both numerical results and the associated figures have proved the credibility of the method.

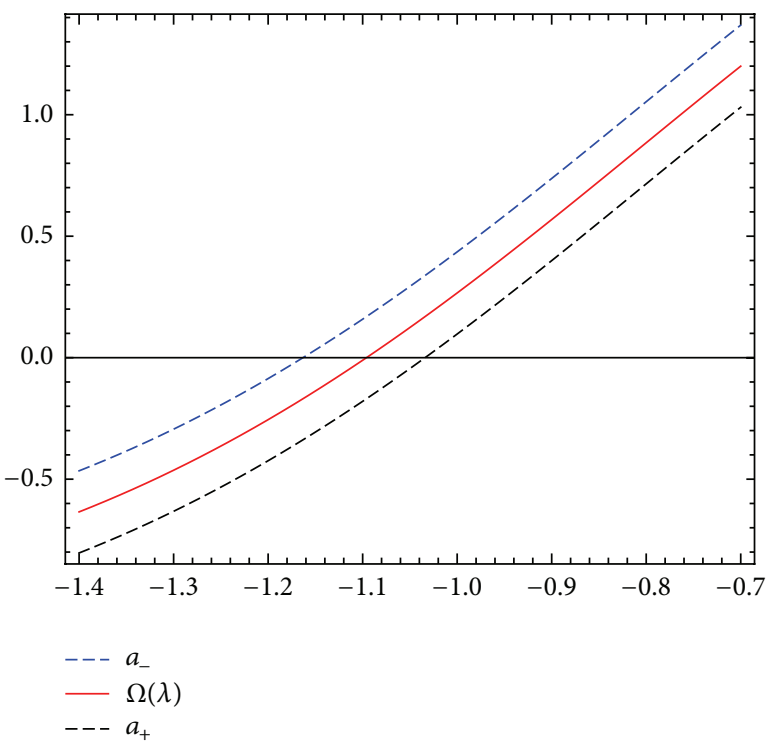

FIGURE 7: The enclosure interval dominating $\lambda_{-1}$ for $h=0.2, N=$ 25 , and $\varepsilon=10^{-2}$.

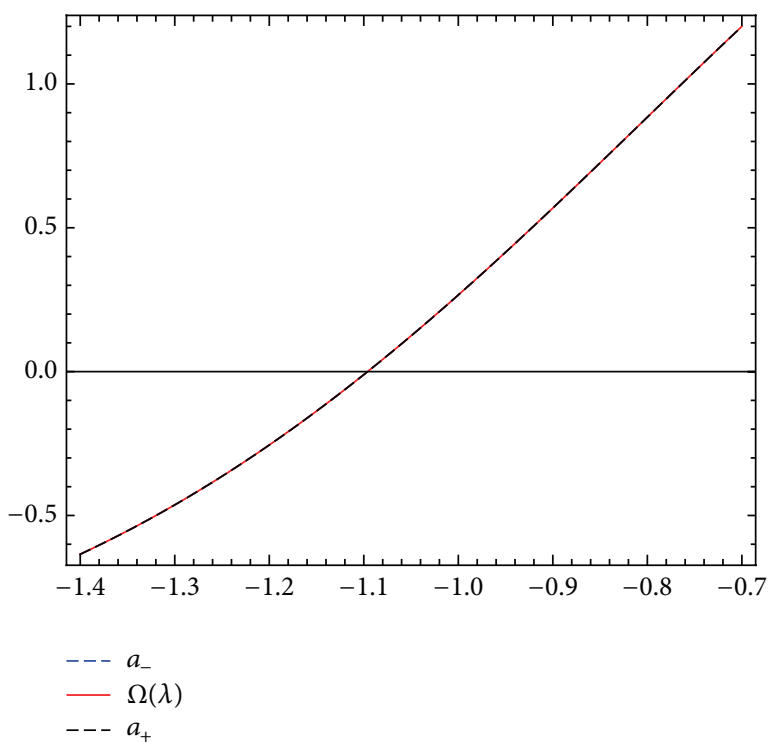

FIGURE 8: The enclosure interval dominating $\lambda_{-1}$ for $h=0.2, N=$ 25 , and $\varepsilon=10^{-5}$.

\section{Conflict of Interests}

The authors declare that there is no conflict of interests regarding the publication of this paper.

\section{Acknowledgment}

This paper was funded by the Deanship of Scientific Research (DSR), King Abdulaziz University, Jeddah. The authors, therefore, acknowledge with thanks DSR technical and financial support. 


\section{References}

[1] C. T. Fulton, "Two-point boundary value problems with eigenvalue parameter contained in the boundary conditions," Proceedings of the Royal Society of Edinburgh A. Mathematics, vol. 77, no. 3-4, pp. 293-308, 1977.

[2] J. Walter, "Regular eigenvalue problems with eigenvalue parameter in the boundary condition," Mathematische Zeitschrift, vol. 133, pp. 301-312, 1973.

[3] V. Kotelnikov, "On the carrying capacity of the ether and wire in telecommunications, material for the first All-Union," in Conference on Questions of Communications, vol. 55, pp. 55-64, Izd. Red. Upr. Svyazi RKKA, 1933.

[4] C. E. Shannon, "Communication in the presence of noise," Proceedings of the IRE, vol. 37, pp. 10-21, 1949.

[5] E. T. Whittaker, "On the functions which are represented by the expansion of the interpolation theory," Proceedings of the Royal Society of Edinburgh A, vol. 35, pp. 181-194, 1915.

[6] P. L. Butzer, G. Schmeisser, and R. L. Stens, "An introduction to sampling analysis," in Non Uniform Sampling: Theory and Practices, F. Marvasti, Ed., pp. 17-121, Kluwer, New York, NY, USA, 2001.

[7] M. A. Kowalski, K. A. Sikorski, and F. Stenger, Selected Topics in Approximation and Computation, Oxford University Press, New York, NY, USA, 1995.

[8] J. Lund and K. L. Bowers, Sinc Methods for Quadrature and Differential Equations, SIAM, Philadelphia, Pa, USA, 1992.

[9] F. Stenger, "Numerical methods based on Whittaker cardinal, or sinc functions," SIAM Review, vol. 23, no. 2, pp. 165-224, 1981.

[10] F. Stenger, Numerical Methods Based on Sinc and Analytic Functions, Springer, New York, NY, USA, 1993.

[11] A. Boumenir, "Higher approximation of eigenvalues by the sampling method," BIT. Numerical Mathematics, vol. 40, no. 2, pp. 215-225, 2000.

[12] A. Boumenir, "Sampling and eigenvalues of non-self-adjoint Sturm-Liouville problems," SIAM Journal on Scientific Computing, vol. 23, no. 1, pp. 219-229, 2001.

[13] M. M. Tharwat, A. H. Bhrawy, and A. Yildirim, "Numerical computation of eigenvalues of discontinuous Sturm-Liouville problems with parameter dependent boundary conditions using sinc method," Numerical Algorithms, vol. 63, no. 1, pp. 2748, 2013.

[14] M. M. Tharwat, A. H. Bhrawy, and A. Yildirim, "Numerical computation of the eigenvalues of a discontinuous Dirac system using the sinc method with error analysis," International Journal of Computer Mathematics, vol. 89, no. 15, pp. 2061-2080, 2012.

[15] P. L. Butzer and R. L. Stens, "A modification of the WhittakerKotel'nikov-Shannon sampling series," Aequationes Mathematicae, vol. 28, no. 3, pp. 305-311, 1985.

[16] R. Gervais, Q. I. Rahman, and G. Schmeisser, "A bandlimited function simulating a duration-limited one," in Approximation Theory and Functional Analysis, P. L. Butzer and R. L. Stens, Eds., pp. 355-362, Birkhäauser, Boston, Mass, USA, 1984.

[17] R. L. Stens, "Sampling by generalized kernels," in Sampling Theory in Fourier and Signal Analysis: Advanced Topics, J. R. Higgins and R. L. Stens, Eds., pp. 130-157, Oxford University Press, Oxford, UK, 1999.

[18] G. Schmeisser and F. Stenger, "Sinc approximation with a Gaussian multiplier," Sampling Theory in Signal and Image Processing, vol. 6, no. 2, pp. 199-221, 2007.
[19] L. Qian, “On the regularized Whittaker-Kotel'nikov-Shannon sampling formula," Proceedings of the American Mathematical Society, vol. 131, no. 4, pp. 1169-1176, 2003.

[20] L. Qian and D. B. Creamer, "A modification of the sampling series with a Gaussian multiplier," Sampling Theory in Signal and Image Processing, vol. 5, no. 1, pp. 1-20, 2006.

[21] L. Qian and D. B. Creamer, "Localized sampling in the presence of noise," Applied Mathematics Letters, vol. 19, no. 4, pp. 351-355, 2006.

[22] M. H. Annaby and R. M. Asharabi, "Computing eigenvalues of boundary-value problems using sinc-Gaussian method," Sampling Theory in Signal and Image Processing, vol. 7, no. 3, pp. 293-312, 2008.

[23] M. H. Annaby and M. M. Tharwat, "A sinc-Gaussian technique for computing eigenvalues of second-order linear pencils," Applied Numerical Mathematics, vol. 63, pp. 129-137, 2013.

[24] A. H. Bhrawy, M. M. Tharwat, and A. Al-Fhaid, "Numerical algorithms for computing eigenvalues of discontinuous Dirac system using sinc-Gaussian method," Abstract and Applied Analysis, vol. 2012, Article ID 925134, 13 pages, 2012.

[25] M. M. Tharwat, A. H. Bhrawy, and A. S. Alofi, "Approximation of eigenvalues of discontinuous sturm-liouville problems with eigenparameter in all boundary conditions," Boundary Value Problems, vol. 2013, article 132, 2013.

[26] M. M. Tharwat and S. M. Al-Harbi, "Approximation of eigenvalues of boundary value problems," Boundary Value Problems, vol. 2014, article 51, 2014.

[27] M. M. Tharwat and A. H. Bhrawy, "Computation of eigenvalues of discontinuous Dirac system using Hermite interpolation technique," Advances in Difference Equations, vol. 2012, article 59, 2012.

[28] M. M. Tharwat, A. Yildirim, and A. H. Bhrawy, "Sampling of discontinuous Dirac systems," Numerical Functional Analysis and Optimization, vol. 34, no. 3, pp. 323-348, 2013.

[29] M. M. Tharwat, "Discontinuous Sturm-Liouville problems and associated sampling theories," Abstract and Applied Analysis, vol. 2011, Article ID 610232, 30 pages, 2011.

[30] M. M. Tharwat, "On sampling theories and discontinuous Dirac systems with eigenparameter in the boundary conditions," Boundary Value Problems, vol. 2013, article 65, 2013.

[31] B. M. Levitan and I. S. Sargsjan, "Introduction to spectral theory: self adjoint ordinary differential operators," in Translation of Mthematical Monographs, vol. 39, American Mathematical Society, Providence, RI, USA, 1975.

[32] B. M. Levitan and I. S. Sargsjan, Sturm-Liouville and Dirac operators, vol. 59, Kluwer Academic Publishers Group, Dordrecht, The Netherlands, 1991. 


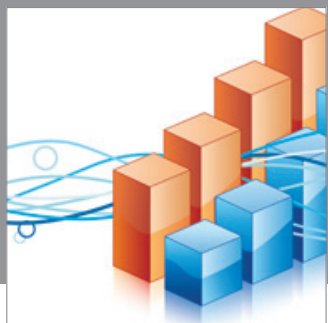

Advances in

Operations Research

mansans

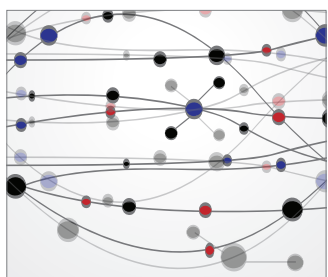

The Scientific World Journal
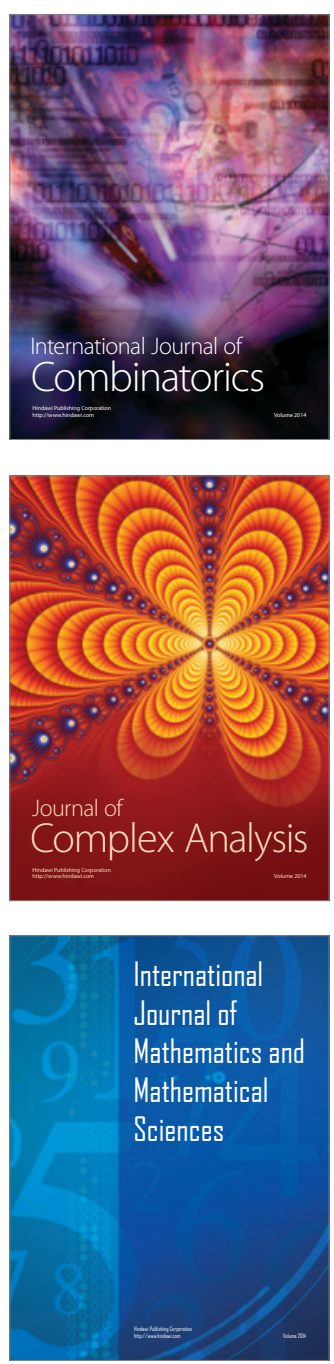
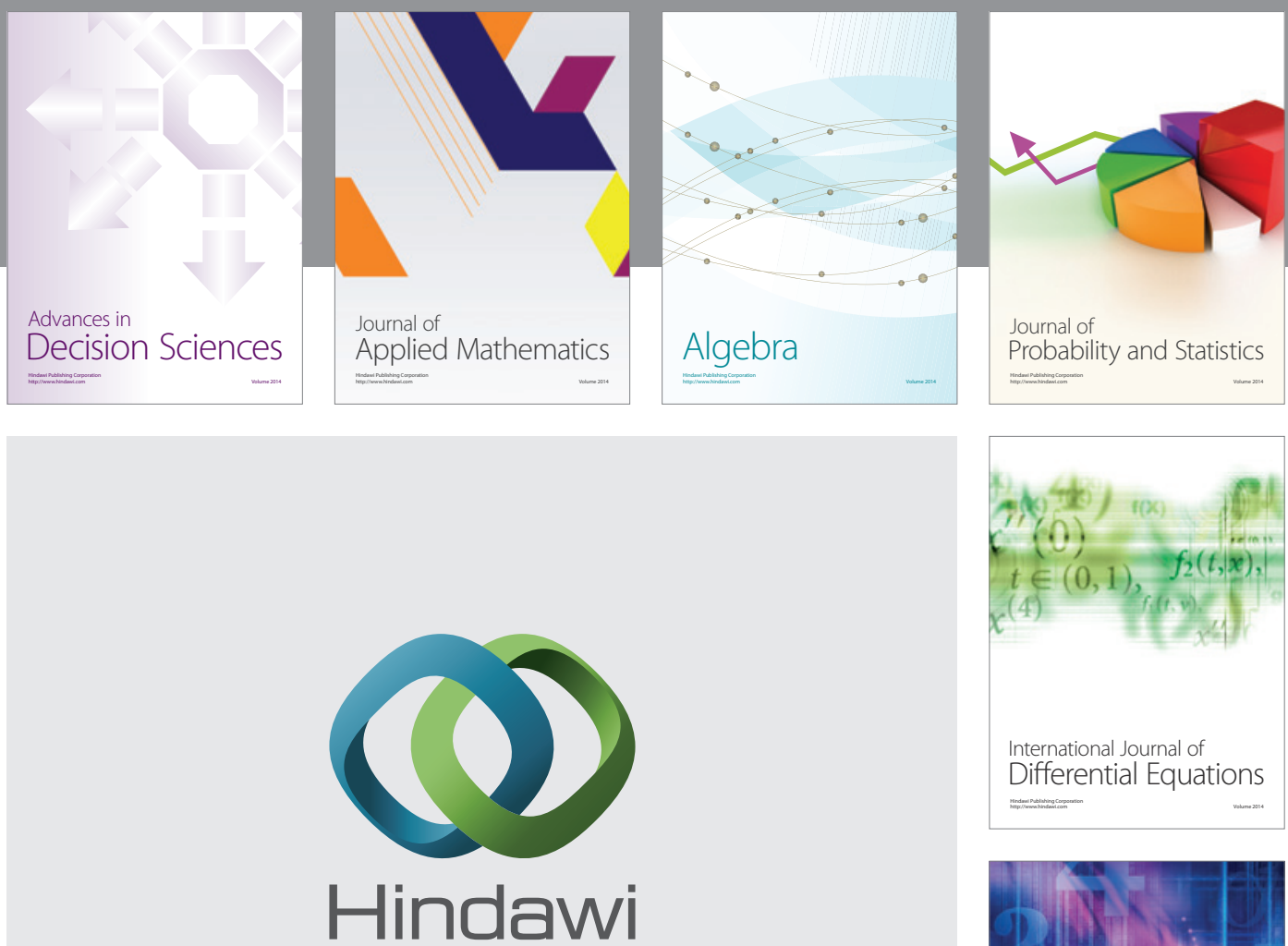

Submit your manuscripts at http://www.hindawi.com
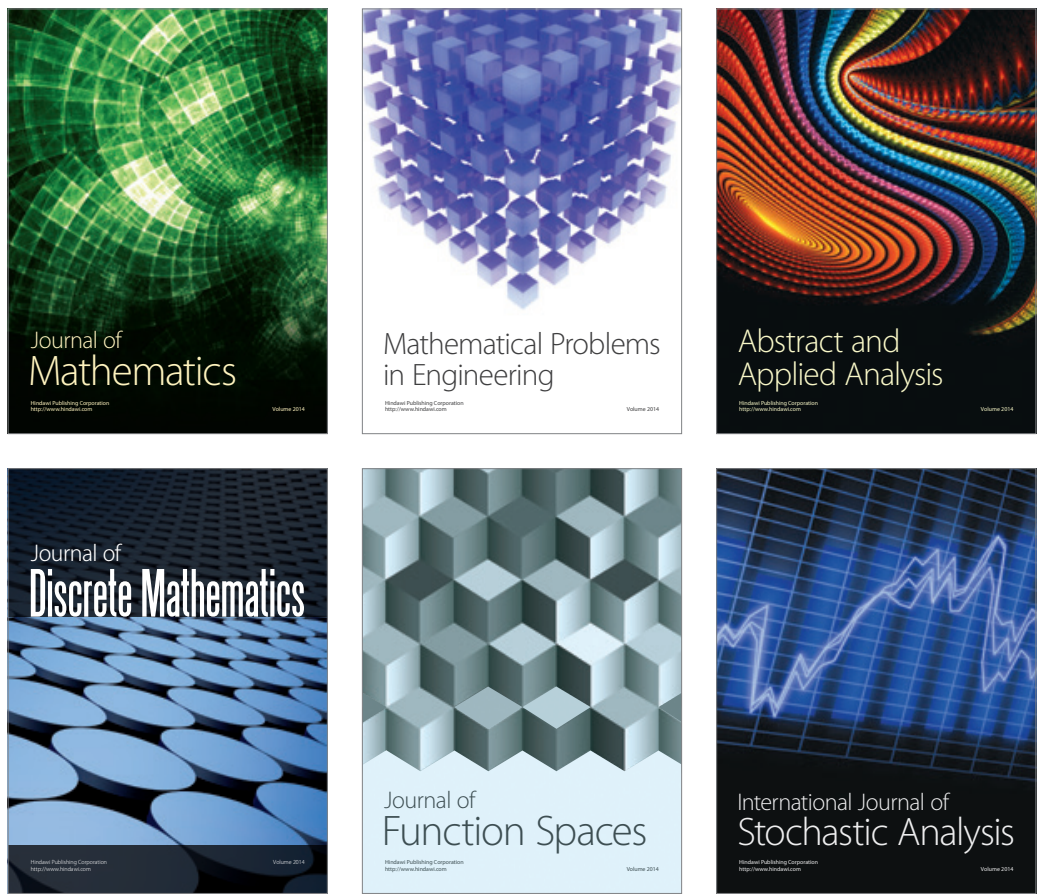

Journal of

Function Spaces

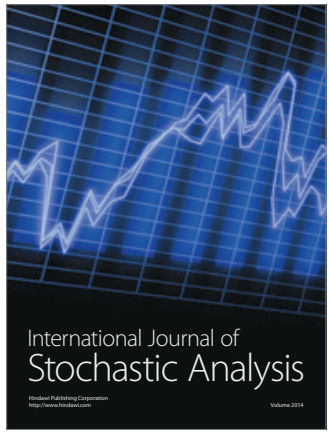

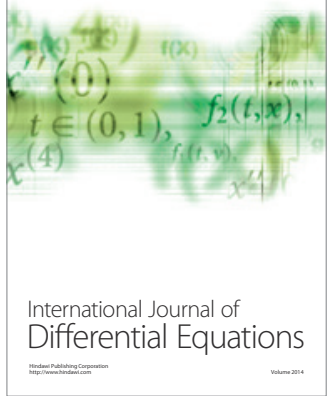
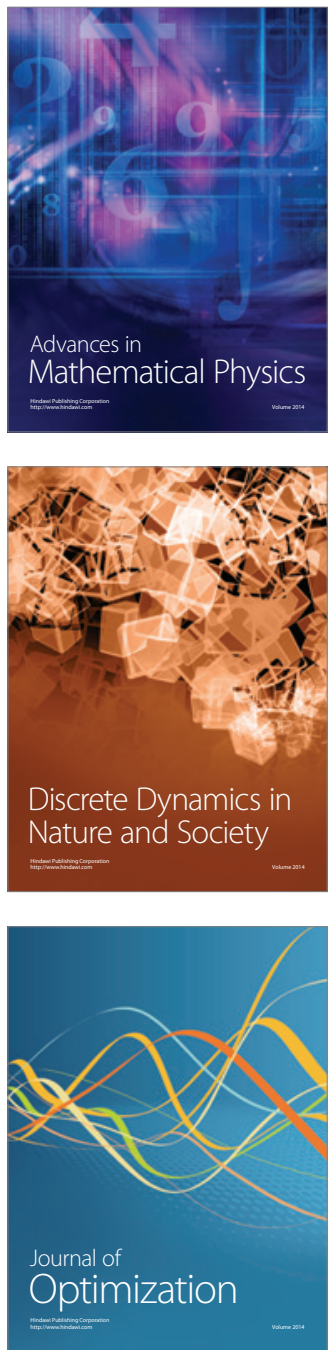